Article

\title{
Ethnomedicinal Uses of Plant Resources in the Machhapuchchhre Rural Municipality of Kaski District, Nepal
}

\author{
Mahendra Adhikari ${ }^{1,2}{ }^{1}$, Rashmi Thapa ${ }^{2}$, Ripu Mardhan Kunwar ${ }^{3}{ }^{(0}$, Hari Prasad Devkota ${ }^{4}(\mathbb{C}$ \\ and Prakash Poudel ${ }^{1, *(D)}$ \\ 1 Department of Pharmacy, Novel Academy, Purbanchal University, Pokhara 33700, Nepal; \\ mahendraadhikari2052@gmail.com \\ 2 School of Health and Allied Sciences, Pokhara University, Pokhara 33700, Nepal; rasmithp@gmail.com \\ 3 Department of Geosciences, Florida Atlantic University, 777 Glades Road, Boca Raton, FL 33431, USA; \\ ripukunwar@gmail.com \\ 4 Graduate School of Pharmaceutical Sciences, Kumamoto University, 5-1 Oe-honmachi, Chuo ku, \\ Kumamoto 862-0973, Japan; devkotah@kumamoto-u.ac.jp \\ * Correspondence: poudelprakesh@gmail.com; Tel.: +977-985-602-9293
}

Received: 31 May 2019; Accepted: 22 June 2019; Published: 23 June 2019

\begin{abstract}
Background: Medicinal plants are being used by the majority of the population throughout the world for their primary health care needs. The reliance is also prevalent in Nepal, aided by its ethnic and biological diversity. This study aimed to catalogue the ethnomedicinal knowledge of plants used by local people of Machhapuchchhre Rural Municipality of Kaski district. Methods: Data were collected between February 2017 and April 2018 from eight different villages of the Kaski district by using semi-structured interviews, guided field works, focus group discussions, and in-depth interviews. The reported traditional uses were supported by local assistants, cataloguing vernacular names and crosschecking with the earlier published and gray literature. Results: A total of 105 medicinal plants, belonging to 58 families and 99 genera were documented to treat 70 different diseases and ailments. The highest numbers of plants (37) were used for gastrointestinal disorders and the lowest (4) were used for female genital disorders. Commonly used parts were underground portions (28 species) followed by fruits and seeds ( 25 species each). The most preferred dosage form was juice, used for 50 ailments, and the oral route was the most favored route of administration ( 77 species). The medicinal properties of 22 plant species were found hitherto unreported in the district. Conclusions: The study area was found to be rich in plant resources and the people have ample knowledge on the use of medicinal plants. Due to a lack of proper documentation, conservation, and cultivation practices, valuable plant species are at risk of extinction. Thus, appropriate conservation measures and scientific assessment of plant-lore in the district is immediately required.
\end{abstract}

Keywords: ethnomedicine; Kaski district; disease and ailments; medicinal plants; Nepal

\section{Introduction}

Traditional medicine has played a crucial role in healthcare system for a long time [1]. The World Health Organization (WHO) defines traditional medicine as the "Sum total of the knowledge, skills, and practices based on the theories, beliefs, and experiences indigenous to different cultures, whether explicable or not, used in the maintenance of health as well as in the prevention, diagnosis, improvement or treatment or physical and mental illness" [2]. Thus, traditional medicine is strongly bonded to nature and dependent on natural resources and culture [3,4]. Historically, plant and animal derived products were the only source of nearly all medicinal preparations [5]. Among them, medicinal plants 
have been the major source of crude drugs used in different traditional medicine systems throughout the world [6-8]. The discovery of the medicinal properties of plants came from long self-experiments, which took centuries for establishment [9]. Due to the lack of appropriate strategies for screening, the use of plant-derived products has diminished in the past two decades [5]. It is estimated that only $10 \%$ of the world's biodiversity has been assessed for their medicinal properties [10]. However, the utilization and determination of their therapeutic potential have not been prioritized to foster the quality of livelihood [11]. In recent years, the application of emerging technology advances in biological activity screening and chemical analysis have increased the interest of researchers towards naturally derived compounds. Lots of active constituents are to be investigated, from both terrestrial and marine sources [5]. In this scenario, the extensive survey of traditionally used plants in their natural habitats will definitely contribute to the identification of medicinally important plants.

In Nepal, it is estimated that only $17 \%$ of people live in urban areas and have access to modern medicine and the rest of the population still depend on the traditional system of medicine for their basic health care needs [12-16]. Traditional healers and elderly people learned folklore through apprenticeships to treat common health disorders based on their ethnomedicinal knowledge [11,17]. However, such knowledge still remains unexplored scientifically [11,13]. Medicinal plant resources have been equally used in the traditional scholarly medical systems, including Ayurveda, Tibetan and Unani, and in folk medicine [18]. Nepal occupies only $0.9 \%$ of the world's land area, but possesses nearly $7 \%$ of the total medicinal herbs of the world $[19,20]$. Over 2000 plant species have been used frequently in rural, remote, and suburban areas of Nepal [21,22]. The uses are associated with the 125 diverse ethnic groups of the country [23]. Some ethnic societies consist of their own healing systems $[7,11]$ and knowledge is transferred orally through generations [7]. However, the local healing system is affected due to changes in lifestyles as a result of globalization, increasing population, land-use change, and global warming. The collection and use of plants substantial for local livelihood, primary health care, and pharmacology [24] has now been severely threatened due to local people's changing perceptions and their context-specific socioeconomic and cultural transformations [25]. Thus, traditional medicine in rural and remote areas of Nepal has experienced a substantial change in the recent decades [26] and it has declined in the absence of proper documentation.

There are a handful of ethnomedicinal studies carried out in Kaski district [27-31], while none is from the rural and remote villages of the Machhapuchchhre rural municipality. The present study, therefore, aims to investigate and document the plant-based indigenous knowledge of local people and explore their traditional uses. We hypothesized that the rural and remote areas of Nepal are rich in medicinal plants and the inhabitants possess unique knowledge of plant use. We also hypothesized that the richness of plants and the knowledge heritage of the area are positively associated.

\section{Materials and Methods}

\subsection{Study Area}

Kaski district lies between $83^{\circ} 40^{\prime}$ east to $84^{\circ} 12^{\prime}$ east longitude and $28^{\circ} 06^{\prime}$ north to $28^{\circ} 36^{\prime}$ north latitude and between $450 \mathrm{~m}$ and $8091 \mathrm{~m}$ elevation above sea level. It comprises a diverse topography (hills, midhills, mountains, Himalaya) and the following five common bio-climates: Sub-tropical, temperate, temperate cold, alpine, and tundra. The average rainfall is about $2500 \mathrm{~mm}$ per annum and the maximum temperature is $34^{\circ} \mathrm{C}$ during the summer (April-July). It is estimated that $46.4 \%$ of the total area of the district is occupied by four types of forest, including subtropical broadleaf forest, temperate forest, subalpine forest, and alpine forest [32]. 


\subsection{Sampling}

The present study was conducted in purposively selected eight remote and rural villages (Koleli, Lwang, Ghalel, Lumre, Sakhu, Siu, Sidhing, and Idikhola) of the Machhapuchchhre rural municipality of the Kaski district (Figure 1). The study villages lie in the Annapurna Conservation Area (ACA), the largest protected area of Nepal [33]. Being located inside the conservation area, the collection of medicinal plants is restricted. The villages are populated with 21,868 inhabitants from 5512 households. The major ethnic groups are Brahmin, Gurung, Kami, Magar, and Chhettri. The communicable language is Nepali but the local people prefer to speak their own dialect, like Gurung and Tamang [32]. Rice, wheat, maize, millet, mustard, potato, etc. are the major crops, while, buffalo, goat, chicken, and cow are the major livestock. Economically, people mostly rely on agriculture and occasional trading of grains, livestock, and cash crops like tea, broom grass, cardamom, Himalayan bamboo shoot, fiddle head fern, and medicinal plants [34]. Although both modern and traditional system of medicines are practiced in this area, there is very limited access to modern allopathic medicine having only one primary health center and seven health posts [32]. Therefore, local people rely on the persistent traditional system of medicine for their basic health care needs.

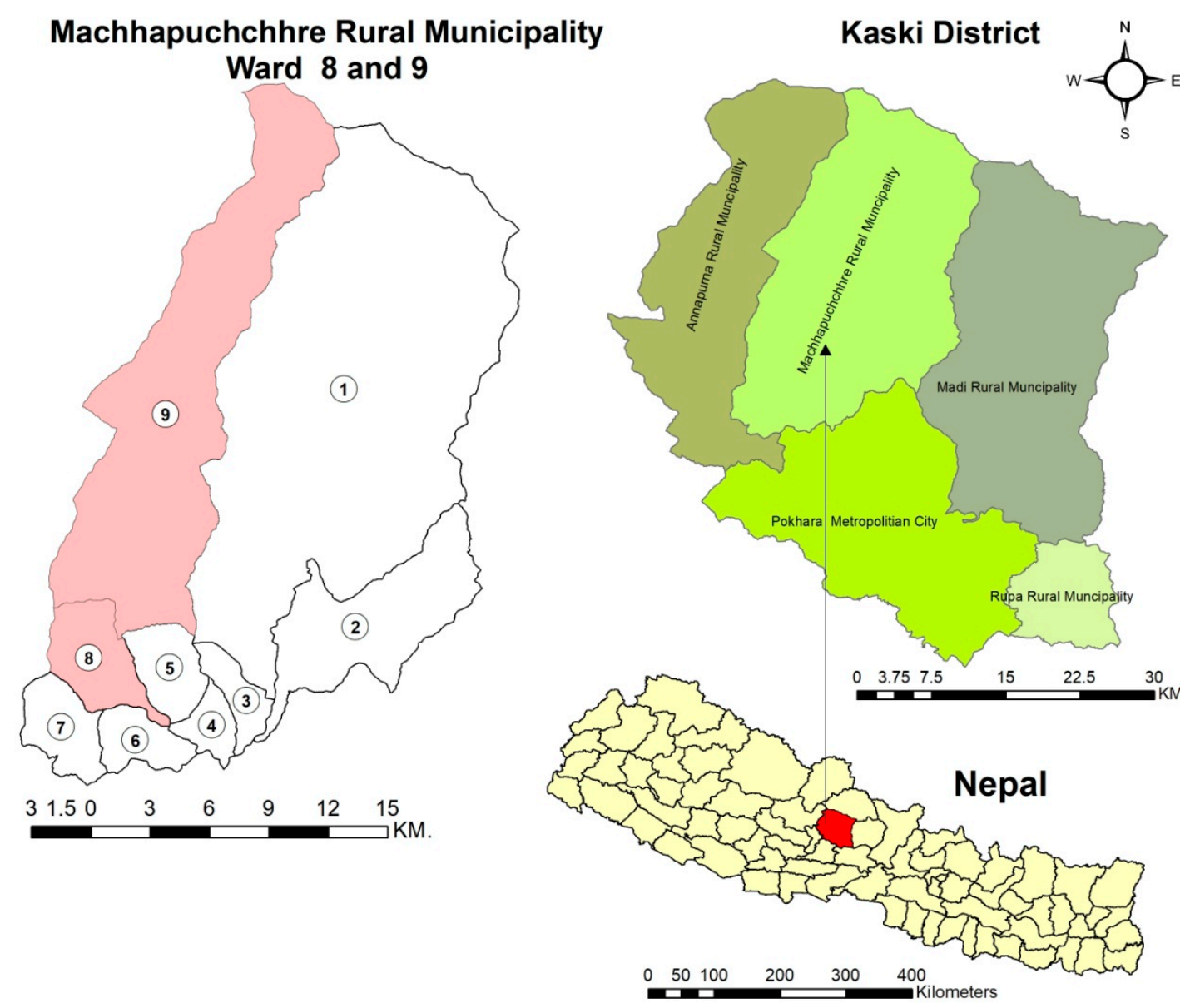

Figure 1. Map of the study area (Machhapuchchhre Rural Municipality) in the Kaski district of Nepal. 


\subsection{Data Collection and Analysis}

The year-long fieldwork was carried between February 2017 and April 2018. A total of 6 field visits were made in each study site. The four main data collection strategies such as Semi-structured interviews, guided field surveys, focus group discussions, and in-depth interviews were used following Martin, 1995 [35]. Ethical approval and prior oral consent was obtained from the Institutional Review Committee (IRC), Pokhara University, Nepal, ACA office and the study participants. During the study, a total of 49 traditional healers including Dhami and Jhankri (11), Pujari (4), Vaidhya (3), and a local herbalist (27) were reached out for study and only 45 allowed us to interview further. Four traditional healers refused to respond to our work because they lamented that the methods of treatment shared among others is disgraceful. A semi-structured questionnaire was designed for the interviews. Among the respondents, $85 \%$ were male and $89 \%$ were above 50 years of age and $72 \%$ were literate. Concerning the ethnicity of participants, Brahmins were found to be dominant (53\%), followed by Gurung (16\%), Lama and Tamang (18\%), and Dalit (13\%).

In situ interviews were taken at the homes of the participants. Questionnaires were explained in Nepali and local dialects. Questions used during the interview sought information including the socio-demographic description of the participants, the local names of plants, plant habitats, parts used, dosage forms, the route of crude drug administration, and disease and ailments treated by the plants. Before conducting extensive interviews, questionnaires were pre-tested with five informants and modified as required. Similarity, dissimilarity, and new indication of plants were confirmed by comparing with previous relevant data $[6,7,11-13,22,27,28,30,31,36-71]$.

The ethnomedicinal uses of plants for various diseases and ailments were analyzed following the International Classification of Primary Care (ICPC) and compared with the literature. The comparison analysis helped to sort out the novelty of the findings. The data were entered in Microsoft Office Excel 2010 to analyze the information regarding plant families, habits, parts used, dosage forms, routes of administration, and the number of ailments treated. Data were expressed in terms of number and percentage. Voucher specimens collected during forest walks were identified using the literature, local vernacular names, and experts' knowledge. Herbarium voucher specimens were cross-identified at the National Herbarium and Plant Laboratories (KATH), Godawari, Lalitpur, Nepal and housed in Novel Academy Herbarium, Novel Academy, Pokhara, Nepal.

\section{Results}

\subsection{Medicinal Plant Diversity and Uses}

Altogether, 105 medicinal plants belonging to 56 families and 99 genera were recorded. Out of 56 families, Asteraceae and Poaceae were the most dominant families, each with 9 species, followed by Fabaceae and Solanaceae (7 species each), Lamiaceae (5 species), Rutaceae (4 species), Amaranthaceae, Apiaceae, and Rosaceae (each with 3 species). The remaining 47 families possessed less than 3 plant species each. The scientific name, family name, local name, and folk uses of the plants are summarized in Table 1. 
Table 1. Enumeration of medicinal plant species used with their parts used, preparation form, local names, family names, mode of application and ailments treated.

\begin{tabular}{|c|c|c|c|c|c|c|c|c|c|}
\hline S.N. & $\begin{array}{l}\text { Botanical Name } \\
\text { Voucher Number }\end{array}$ & Family & Local Name & Habit & Parts Used & Preparation & $\begin{array}{l}\text { Mode of } \\
\text { Application }\end{array}$ & Ailments Treated/ Uses & $\begin{array}{l}\text { Previous Uses } \\
\text { Reported References }\end{array}$ \\
\hline 1. & $\begin{array}{l}\text { Abelmoschus manihot var. } \\
\text { pungens Hochr. } \\
\text { NAH-25 }\end{array}$ & Malvaceae & Kapasi & Herb & Root & $\begin{array}{l}\text { Decoction and } \\
\text { juice }\end{array}$ & Oral & Fever & [72] \\
\hline \multirow[t]{2}{*}{2.} & \multirow{2}{*}{$\begin{array}{l}\text { Achyranthes bidentata Bl. } \\
\text { NAH-75 }\end{array}$} & \multirow{2}{*}{ Amaranthaceae } & \multirow{2}{*}{ Datiwan } & \multirow{2}{*}{ Herb } & Root & Juice & Oral & $\begin{array}{l}\text { Typhoid, Tonsillitis, } \\
\text { Urine retention }\end{array}$ & \multirow{2}{*}[7,36,39,40,59]{} \\
\hline & & & & & Stem & Powder & Chewing & Toothache & \\
\hline 3. & $\begin{array}{l}\text { Acmella calva (DC.) R.K. Jansen } \\
\text { NAH-50 }\end{array}$ & Asteraceae & Marathi & Herb & Fruit & Pickle & Oral & $\begin{array}{l}\text { Intestinal worm, Gastritis, } \\
\text { Flatulence }\end{array}$ & [69] \\
\hline 4. & $\begin{array}{l}\text { Acorus calamus L. } \\
\text { NAH-35 }\end{array}$ & Acoraceae & Bojho & Herb & Rhizome & $\begin{array}{l}\text { Chewable } \\
\text { (rhizome) }\end{array}$ & Oral & $\begin{array}{l}\text { Cough, Chest pain, } \\
\text { Asthma }\end{array}$ & $\begin{array}{l}{[13,22,31,36,40,44,46,} \\
47,49,52,53,57,58,63, \\
69,71,73]\end{array}$ \\
\hline 5. & $\begin{array}{l}\text { Ageratina adenophora (Spreng.) } \\
\text { R. M. King \& H. Rob. } \\
\text { NAH-52 }\end{array}$ & Asteraceae & Banmara & Shrub & Leaf & Juice & Topical & $\begin{array}{l}\text { Bleeding, Cuts and } \\
\text { Wounds }\end{array}$ & None \\
\hline 6. & $\begin{array}{l}\text { Aleuritopteris bicolor (Roxb.) } \\
\text { Fraser-Jenk. } \\
\text { NAH-55 }\end{array}$ & Pteridaceae & Dankernu & Fern & Whole plant & Juice & Oral & $\begin{array}{l}\text { Diarrhea, Dysentery, } \\
\text { Gastritis }\end{array}$ & None \\
\hline \multirow[t]{2}{*}{7.} & \multirow{2}{*}{$\begin{array}{l}\text { Allium sativum L. } \\
\text { NAH-112 }\end{array}$} & \multirow[t]{2}{*}{ Amaryllidaceae } & \multirow[t]{2}{*}{ Lasun } & \multirow[t]{2}{*}{ Herb } & Bulb & Decoction & Oral & $\begin{array}{l}\text { Gastritis, Flatulence, } \\
\text { Diarrhea, Dysentery }\end{array}$ & \multirow[t]{2}{*}[6,7,59,65,70]{} \\
\hline & & & & & Bulb & Paste & Topical & Snakebite & \\
\hline 8. & $\begin{array}{l}\text { Aloe vera (L.) Burm.f. } \\
\text { NAH-60 }\end{array}$ & Xanthorrhoeaceae & Gheukumari & Herb & Leaf & Gel & Topical & Burns, Boils & {$[28,36,40,44,66,70]$} \\
\hline \multirow{2}{*}{9.} & \multirow{2}{*}{$\begin{array}{l}\text { Alternanthera sessilis (L.) R. Br. } \\
\text { Ex DC. } \\
\text { NAH-133 }\end{array}$} & \multirow{2}{*}{ Amaranthaceae } & \multirow{2}{*}{ Bhiringi Jhar } & \multirow{2}{*}{ Herb } & Leaf & Juice & Topical & Bleeding & \multirow{2}{*}[11,44,52,69]{} \\
\hline & & & & & Leaf & Juice and paste & Topical & Cuts and Wounds & \\
\hline 10. & $\begin{array}{l}\text { Anaphalis triplinervis (Sims) } \\
\text { Sims ex C.B. Clarke } \\
\text { NAH-120 }\end{array}$ & Asteraceae & Buki ful & Herb & Whole plant & Juice & Oral & Post-partum hemorrhage & {$[41,60,74,75]$} \\
\hline 11. & $\begin{array}{l}\text { Artemisia dubia L. ex B. D. Jacks. } \\
\text { NAH-110 }\end{array}$ & Asteraceae & Tite pati & Herb & Leaf & Juice and paste & Topical & Scabies & {$[11,36,46,50,57,74,76]$} \\
\hline 12. & $\begin{array}{l}\text { Asparagus racemosus Willd. } \\
\text { NAH-109 }\end{array}$ & Asperagaceae & $\begin{array}{l}\text { Kurilo/ } \\
\text { Satawari }\end{array}$ & Shrub & Whole plant & $\begin{array}{l}\text { Juice and } \\
\text { decoction }\end{array}$ & Oral & $\begin{array}{l}\text { Hypertension, Diabetes } \\
\text { mellitus }\end{array}$ & $\begin{array}{l}{[13,27,30,31,36,39-41,} \\
44,47-49,53,57,59,63, \\
71,77,78]\end{array}$ \\
\hline
\end{tabular}


Table 1. Cont

\begin{tabular}{|c|c|c|c|c|c|c|c|c|c|}
\hline S.N. & $\begin{array}{l}\text { Botanical Name } \\
\text { Voucher Number }\end{array}$ & Family & Local Name & Habit & Parts Used & Preparation & $\begin{array}{l}\text { Mode of } \\
\text { Application }\end{array}$ & Ailments Treated/ Uses & $\begin{array}{l}\text { Previous Uses } \\
\text { Reported References }\end{array}$ \\
\hline 13. & $\begin{array}{l}\text { Azadirachta indica A. Juss } \\
\text { NAH-121 }\end{array}$ & Meliaceae & Neem & Tree & Bark & Juice & Oral & Fever, Skin disorders & $\begin{array}{l}{[11,28,36,38-40,44,49,} \\
52,53,76,79]\end{array}$ \\
\hline 14. & $\begin{array}{l}\text { Bauhinia malabarica Roxb. } \\
\text { NAH-67 }\end{array}$ & Fabaceae & Tanki & Tree & $\begin{array}{l}\text { Bark and } \\
\text { root }\end{array}$ & $\begin{array}{l}\text { Juice and } \\
\text { decoction }\end{array}$ & Oral & Typhoid, tonsillitis & None \\
\hline 15. & $\begin{array}{l}\text { Bauhinia purpurea L. } \\
\text { NAH-01 }\end{array}$ & Fabaceae & Koiralo & Tree & Bark & Decoction & Oral & Gastritis & {$[36,69]$} \\
\hline 16. & $\begin{array}{l}\text { Berberis aristata DC. } \\
\text { NAH-124 }\end{array}$ & Berberidaceae & Chutro & Shrub & $\begin{array}{l}\text { Bark and } \\
\text { root }\end{array}$ & Juice & Oral & $\begin{array}{l}\text { Typhoid, Fever, Diarrhea, } \\
\text { Jaundice }\end{array}$ & $\begin{array}{l}{[6,13,22,30,31,36,40-} \\
42,46,50-52,57,60,63 \\
69,71,73-75,77,80]\end{array}$ \\
\hline \multirow[b]{2}{*}{17.} & \multirow[b]{2}{*}{$\begin{array}{l}\text { Bergenia ciliata (Haw.) Sternb. } \\
\text { NAH-36 }\end{array}$} & \multirow[b]{2}{*}{ Saxifragaceae } & \multirow[b]{2}{*}{ Pakhanbed } & \multirow[b]{2}{*}{ Herb } & Rhizome & Paste & Topical & Sprain, Fracture & \multirow{2}{*}{$\begin{array}{l}{[7,13,22,36,37,40,42,} \\
50-52,56,57,59,60,63 \\
66,70,73-80]\end{array}$} \\
\hline & & & & & Rhizome & $\begin{array}{l}\text { Powder along } \\
\text { with rice flour } \\
\text { in ghee }\end{array}$ & Oral & Body ache, Backache & \\
\hline 18. & $\begin{array}{l}\text { Betula alnoides Buch.-Ham.ex D. } \\
\text { Don } \\
\text { NAH-22 }\end{array}$ & Betulaceae & Saur & Tree & Bark & Juice & Oral & Fever, Backache & {$[7,63,73,78]$} \\
\hline 19. & $\begin{array}{l}\text { Bryophyllum pinnatum (Lam.) } \\
\text { Oken } \\
\text { NAH-14 }\end{array}$ & Crassulaceae & Ajambari & Herb & Flower & Juice & Aural & Earache & {$[69,79]$} \\
\hline 20. & $\begin{array}{l}\text { Centella asiatica (L.) Urb. } \\
\text { NAH-104 }\end{array}$ & Apiaceae & Ghod tapre & Herb & Whole plant & Paste and juice & Oral & $\begin{array}{l}\text { Typhoid, Cough, } \\
\text { Tonsillitis, Gastritis }\end{array}$ & $\begin{array}{l}{[11,27,30,31,36,39,41} \\
42,44,46,47,49,53,57- \\
59,63,69,71,79]\end{array}$ \\
\hline 21. & $\begin{array}{l}\text { Chenopodium album } \mathrm{L} \text {. } \\
\text { NAH-12 }\end{array}$ & Amaranthaceae & Bethe & Herb & $\begin{array}{l}\text { Leaf and } \\
\text { fruit }\end{array}$ & $\begin{array}{l}\text { Juice and } \\
\text { decoction }\end{array}$ & Oral & $\begin{array}{l}\text { Gastritis, Flatulence, } \\
\text { Stomachache }\end{array}$ & {$[22,36,38,53,57,65]$} \\
\hline 22. & $\begin{array}{l}\text { Cinnamomum tamala } \\
\text { (Buch.-Ham.) T. Nees \& Eberm } \\
\text { NAH-107 }\end{array}$ & Lauraceae & $\begin{array}{l}\text { Tejpat/ } \\
\text { Dalchini }\end{array}$ & Tree & Leaf & Decoction & Oral & $\begin{array}{l}\text { Fever, Stomachache, } \\
\text { Gastritis }\end{array}$ & $\begin{array}{l}{[31,36,39,40,47,50,57,} \\
58,63,73,80]\end{array}$ \\
\hline \multirow{2}{*}{23.} & \multirow{2}{*}{$\begin{array}{l}\text { Cirsium verutum (D. Don) } \\
\text { Spreng. } \\
\text { NAH-70 }\end{array}$} & \multirow{2}{*}{ Asteraceae } & \multirow{2}{*}{$\begin{array}{l}\text { Thakailo/ } \\
\text { Thakali kanda }\end{array}$} & \multirow{2}{*}{ Herb } & $\begin{array}{l}\text { Bud and } \\
\text { root }\end{array}$ & $\begin{array}{l}\text { Chewable (bud } \\
\text { and root) }\end{array}$ & Oral & $\begin{array}{l}\text { Sore throat, Nose } \\
\text { bleeding }\end{array}$ & \multirow{2}{*}[30,42,45,57,63,76]{} \\
\hline & & & & & Root & Juice & Oral & $\begin{array}{l}\text { Typhoid, Diabetes } \\
\text { mellitus }\end{array}$ & \\
\hline 24. & $\begin{array}{l}\text { Cissampelos pareira } \mathrm{L} \text {. } \\
\text { NAH-71 }\end{array}$ & Menispermacae & $\begin{array}{l}\text { Thulo Batul } \\
\text { pate }\end{array}$ & Climber & Whole plant & Decoction & Oral & Postpartum hemorrhage & $\begin{array}{l}{[11,28,36,41,44,47,52} \\
57,59,69,74,80]\end{array}$ \\
\hline
\end{tabular}


Table 1. Cont

\begin{tabular}{|c|c|c|c|c|c|c|c|c|c|}
\hline S.N. & $\begin{array}{l}\text { Botanical Name } \\
\text { Voucher Number }\end{array}$ & Family & Local Name & Habit & Parts Used & Preparation & $\begin{array}{l}\text { Mode of } \\
\text { Application }\end{array}$ & Ailments Treated/ Uses & $\begin{array}{l}\text { Previous Uses } \\
\text { Reported References }\end{array}$ \\
\hline 25. & $\begin{array}{l}\text { Citrus medica L. } \\
\text { NAH-11 }\end{array}$ & Rutaceae & Bimiro & Tree & $\begin{array}{l}\text { Root and } \\
\text { fruit }\end{array}$ & Juice & Oral & Intestinal worms & [80] \\
\hline 26. & $\begin{array}{l}\text { Citrus aurantiifolia (Christm.) } \\
\text { Swingle } \\
\text { NAH-93 }\end{array}$ & Rutaceae & Kagati & Tree & Fruit & $\begin{array}{l}\text { Juice, juice with } \\
\text { honey }\end{array}$ & Oral & $\begin{array}{l}\text { Thirst, Jaundice, } \\
\text { Anorexia, Pimple, Skin } \\
\text { disorders }\end{array}$ & {$[7,39,58,59]$} \\
\hline 27. & $\begin{array}{l}\text { Citrus limon (L.) Osbeck } \\
\text { NAH-59 }\end{array}$ & Rutaceae & Nibuwa & Tree & Fruit & Juice & Oral & $\begin{array}{l}\text { Food poisoning, Blood } \\
\text { purification }\end{array}$ & {$[11,39,48,53,65,66,76]$} \\
\hline 28. & $\begin{array}{l}\text { Coelogyne cristata Lindl. } \\
\text { NAH-122 }\end{array}$ & Orchidaceae & Kadam & Orchid & Pseudobulb & $\begin{array}{l}\text { Powder mixed } \\
\text { with cooked rice } \\
\text { flour in butter } \\
\text { (Puwa) }\end{array}$ & Oral & $\begin{array}{l}\text { Backache, Fracture, } \\
\text { Sprain }\end{array}$ & None \\
\hline 29. & $\begin{array}{l}\text { Coffea Arabica L. } \\
\text { NAH-63 }\end{array}$ & Rubiaceae & Kafi & Shrub & Fruit & Decoction & Oral & Lethargy, Headache & [64] \\
\hline \multirow[b]{2}{*}{30.} & \multirow[b]{2}{*}{$\begin{array}{l}\text { Colocasia esculenta (L.) Schott } \\
\text { NAH-20 }\end{array}$} & \multirow[b]{2}{*}{ Araceae } & \multirow[b]{2}{*}{ Karkalo } & \multirow[b]{2}{*}{ Herb } & Stem & Latex & Topical & Snakebite & \multirow[b]{2}{*}[30,40,53]{} \\
\hline & & & & & $\begin{array}{l}\text { Whole plant } \\
\text { and root }\end{array}$ & $\begin{array}{l}\text { Decoction and } \\
\text { vegetable curry }\end{array}$ & Oral & Constipation & \\
\hline 31. & $\begin{array}{l}\text { Cucurbita pepo L. } \\
\text { NAH-26 }\end{array}$ & Cucurbitaceae & Farsi & Herb & Fruit & Boiledfruit & Oral & Jaundice, Gastritis & {$[22,64,66]$} \\
\hline 32. & $\begin{array}{l}\text { Curcuma longa L. } \\
\text { NAH-111 }\end{array}$ & Zingiberaceae & Besar/Haledo & Herb & Rhizome & Decoction & Oral & $\begin{array}{l}\text { Fever, Sore throat, } \\
\text { Sinusitis, Common cold }\end{array}$ & $\begin{array}{l}{[31,38,52,53,58,59,75,} \\
80]\end{array}$ \\
\hline 33. & $\begin{array}{l}\text { Cuscuta reflexa Roxb. } \\
\text { NAH-92 }\end{array}$ & Convolvulaceae & Aakase beli & Herb & Whole plant & Juice & Oral & Jaundice & $\begin{array}{l}{[11,22,31,39-41,43,44,} \\
47,49,51-53,59,63]\end{array}$ \\
\hline 34. & $\begin{array}{l}\text { Cyathea spinulosa Wall. ex } \\
\text { Hook. } \\
\text { NAH-32 }\end{array}$ & Cyatheaceae & Chattre & Fern & Soft pith & $\begin{array}{l}\text { Decoction } \\
\text { prepared in } \\
\text { ghee }\end{array}$ & Oral & Fracture, Body ache & [30] \\
\hline 35. & $\begin{array}{l}\text { Cynodon dactylon (L.) Pers. } \\
\text { NAH-74 }\end{array}$ & Poaceae & Dubo & Grass & Whole plant & Juice & Oral & $\begin{array}{l}\text { Diarrhea, Dysentery, } \\
\text { Intestinal worm, } \\
\text { Flatulence }\end{array}$ & $\begin{array}{l}{[6,11,39,44,45,49,51} \\
53,57,58,63,70,73,80]\end{array}$ \\
\hline 36. & $\begin{array}{l}\text { Dactylicapnos macrocapnos } \\
\text { (Prain) Hutch. } \\
\text { NAH-16 }\end{array}$ & Papaveraceae & $\begin{array}{l}\text { Bichkane } \\
\text { Jhar }\end{array}$ & Climber & Whole plant & Squeezed Juice & Topical & Corneal scar & None \\
\hline 37. & $\begin{array}{l}\text { Daucus carota } \mathrm{L} \text {. } \\
\text { NAH-72 }\end{array}$ & Apiaceae & Gajar & Herb & Rhizome & $\begin{array}{l}\text { Chewable } \\
\text { (rhizome) }\end{array}$ & Oral & Jaundice, Blindness & {$[39,56,65]$} \\
\hline
\end{tabular}


Table 1. Cont

\begin{tabular}{|c|c|c|c|c|c|c|c|c|c|}
\hline S.N. & $\begin{array}{l}\text { Botanical Name } \\
\text { Voucher Number }\end{array}$ & Family & Local Name & Habit & Parts Used & Preparation & $\begin{array}{l}\text { Mode of } \\
\text { Application }\end{array}$ & Ailments Treated/ Uses & $\begin{array}{l}\text { Previous Uses } \\
\text { Reported References }\end{array}$ \\
\hline 38. & $\begin{array}{l}\text { Desmostachya bipinnata (L.) } \\
\text { Stapf. } \\
\text { NAH-04 }\end{array}$ & Poaceae & Kush & Grass & Root & Paste & Topical & Toothache & {$[40,63]$} \\
\hline 39. & $\begin{array}{l}\text { Dinetus racemosus (Roxb.) } \\
\text { Buch.-Ham. ex Sweet } \\
\text { NAH-62 }\end{array}$ & Convolvulaceae & Badimal & Climber & Rhizome & Decoction & Oral & Post-partum hemorrhage & None \\
\hline 40. & $\begin{array}{l}\text { Dioscorea belophylla (Prain) } \\
\text { Voight ex Haines } \\
\text { NAH-07 }\end{array}$ & Dioscoreaceae & Ban Tarul & Climber & Tuber & Boiled tuber & Oral & Constipation & None \\
\hline 41. & $\begin{array}{l}\text { Drepanostachyum falcatum } \\
\text { (Nees) Keng f. } \\
\text { NAH-34 }\end{array}$ & Poaceae & Nigalo & Grass & Stem & $\begin{array}{l}\text { Dust outside the } \\
\text { stem }\end{array}$ & Topical & Tinea pedis & {$[7,30]$} \\
\hline \multirow[t]{2}{*}{42.} & \multirow{2}{*}{$\begin{array}{l}\text { Drymaria diandra Blume } \\
\text { NAH-138 }\end{array}$} & \multirow[t]{2}{*}{ Caryophyllaceae } & \multirow[t]{2}{*}{ Abijalo } & \multirow{2}{*}{ Herb } & \multirow[t]{2}{*}{ Whole plant } & Paste and juice & Oral & $\begin{array}{l}\text { Gastritis, Flatulence, } \\
\text { Nausea, Vomiting }\end{array}$ & \multirow{2}{*}{$\begin{array}{l}{[28,39,51-53,58,63,78,} \\
80]\end{array}$} \\
\hline & & & & & & Stem vapor & Inhalation & Sinusitis & \\
\hline 43. & $\begin{array}{l}\text { Eclipta prostrata (L.) L. } \\
\text { NAH-61 }\end{array}$ & Asteraceae & Bhiringe & Herb & Leaf & Juice & Topical & Bleeding, Wound & {$[11,27,47,52,53,69,78]$} \\
\hline 44. & $\begin{array}{l}\text { Eleusine coracana (L.) Gaertn. } \\
\text { NAH-30 }\end{array}$ & Poaceae & Kodo & Grass & Fruit & Cooked flour & Oral & Cough & [69] \\
\hline 45. & $\begin{array}{l}\text { Emilia sonchifolia (L.) DC. ex } \\
\text { DC. } \\
\text { NAH-10 }\end{array}$ & Asteraceae & Salha ko jhar & Herb & Whole plant & Juice & Topical & $\begin{array}{l}\text { Bleeding, Cuts and } \\
\text { Wounds }\end{array}$ & [11] \\
\hline 46. & $\begin{array}{l}\text { Engelhardia spicata Lesch. ex } \\
\text { Blume } \\
\text { NAH-77 }\end{array}$ & Juglandaceae & Mauwa & Tree & Bud & Paste & Topical & Tinea pedis & {$[28,39,58,80]$} \\
\hline 47. & $\begin{array}{l}\text { Erythrina stricta Roxb. } \\
\text { NAH-23 }\end{array}$ & Fabaceae & Fadelo & Tree & Bark & $\begin{array}{l}\text { Juice and } \\
\text { decoction }\end{array}$ & Oral & Typhoid, Sore throat & [78] \\
\hline 48. & $\begin{array}{l}\text { Eurya acuminata DC. } \\
\text { NAH-118 }\end{array}$ & Pentaphylacaceae & Jhyanu & Shrub & Bud & Juice & Oral & $\begin{array}{l}\text { Typhoid, Tonsillitis, Sore } \\
\text { throat }\end{array}$ & None \\
\hline 49. & $\begin{array}{l}\text { Ficus religiosa } \mathrm{L} \text {. } \\
\text { NAH- } 56\end{array}$ & Moraceae & Pipal & Tree & Leaf & Juice & Topical & Cuts and Wounds & $\begin{array}{l}{[11,36,38,40,49,57,63,} \\
66,69]\end{array}$ \\
\hline 50. & $\begin{array}{l}\text { Foeniculum vulgare Mill. } \\
\text { NAH-99 }\end{array}$ & Apiaceae & Sonp & Herb & Fruit & $\begin{array}{l}\text { Powder mixed } \\
\text { with cooked rice } \\
\text { flour in butter } \\
\text { (Puwa) }\end{array}$ & Oral & Bone weakness, Fracture & {$[56,64,81]$} \\
\hline
\end{tabular}


Table 1. Cont

\begin{tabular}{|c|c|c|c|c|c|c|c|c|c|}
\hline S.N. & $\begin{array}{l}\text { Botanical Name } \\
\text { Voucher Number }\end{array}$ & Family & Local Name & Habit & Parts Used & Preparation & $\begin{array}{l}\text { Mode of } \\
\text { Application }\end{array}$ & Ailments Treated/ Uses & $\begin{array}{l}\text { Previous Uses } \\
\text { Reported References }\end{array}$ \\
\hline 51. & $\begin{array}{l}\text { Gonostegia hirta (Blume ex } \\
\text { Hassk.) Miq. } \\
\text { NAH-08 }\end{array}$ & Urticaceae & Aaternu & Herb & Leaf & Paste & Topical & Boils & None \\
\hline 52. & $\begin{array}{l}\text { Hoya lanceolata Wall. ex D. Don } \\
\text { NAH-09 }\end{array}$ & Asclepiadaceae & Thirjo & Shrub & Whole plant & Juice & Oral & Body ache & None \\
\hline 53. & $\begin{array}{l}\text { Imperata cylindrica (L.) Raeusch. } \\
\text { NAH- } 45\end{array}$ & Poaceae & Siru & Grass & Root & Juice & Oral & Typhoid & {$[22,38,57,63]$} \\
\hline \multirow[t]{2}{*}{54.} & \multirow{2}{*}{$\begin{array}{l}\text { Jatropha curcas L. } \\
\text { NAH-38 }\end{array}$} & Euphorbiaceae & Sajiwan & Tree & $\begin{array}{l}\text { Leaf and } \\
\text { stem }\end{array}$ & Juice and paste & Gargling & $\begin{array}{l}\text { Gingivitis, Tonsillitis, } \\
\text { Sore throat }\end{array}$ & \multirow[t]{2}{*}[11,36,44,47,57,63]{} \\
\hline & & & & & & Latex & Topical & Fungal infection & \\
\hline 55. & $\begin{array}{l}\text { Juglans regia L. } \\
\text { NAH-27 }\end{array}$ & Juglandaceae & Okhar & Tree & Peels of fruit & Paste & Topical & $\begin{array}{l}\text { Tinea pedis, Fungal } \\
\text { infection }\end{array}$ & $\begin{array}{l}{[13,31,37,46,47,58,60} \\
63,71,74,75,77]\end{array}$ \\
\hline 56. & $\begin{array}{l}\text { Linum usitatissimum L. } \\
\text { NAH-88 }\end{array}$ & Linaceae & Aatasi & Herb & Seed & Roast & Oral & $\begin{array}{l}\text { Lethargy, Intestinal } \\
\text { worms }\end{array}$ & [53] \\
\hline \multirow{2}{*}{57.} & \multirow{2}{*}{$\begin{array}{l}\text { Litsea cubeba (Lour.) Pers. } \\
\text { NAH- } 40\end{array}$} & \multirow{2}{*}{ Lauraceae } & \multirow{2}{*}{ Siltimur } & \multirow{2}{*}{ Tree } & Seed & $\begin{array}{l}\text { Decoction and } \\
\text { spice }\end{array}$ & Oral & $\begin{array}{l}\text { Gastritis, Flatulence, } \\
\text { Indigestion, Gastric } \\
\text { troubles }\end{array}$ & \multirow{2}{*}[31,40]{} \\
\hline & & & & & Bark & Paste & Topical & $\begin{array}{l}\text { Cuts and Wounds, } \\
\text { Fracture }\end{array}$ & \\
\hline 58. & $\begin{array}{l}\text { Lycopersicon esculentum Mill. } \\
\text { NAH-57 }\end{array}$ & Solanaceae & Golveda & Herb & Fruit & Juice & Oral & Burns, Boils & {$[39,65,80]$} \\
\hline 59. & $\begin{array}{l}\text { Lyonia ovalifolia (Wall.) Drude } \\
\text { NAH-94 }\end{array}$ & Ericaceae & Thaune & Tree & Bud & Paste & Topical & Scabies & {$[36,39,41,58,59,63]$} \\
\hline 60. & $\begin{array}{l}\text { Macaranga pustulata King ex } \\
\text { Hook.f. } \\
\text { NAH-19 }\end{array}$ & Euphorbiaceae & Mallato & Tree & Stem & Latex & Topical & Boils & None \\
\hline 61. & $\begin{array}{l}\text { Maesa chisia Buch. Ham. ex. D. } \\
\text { Don } \\
\text { NAH-02 }\end{array}$ & Primulaceae & Bilaune & Shrub & $\begin{array}{l}\text { Fruit and } \\
\text { Root }\end{array}$ & Juice and paste & Topical & Scabies & None \\
\hline 62. & $\begin{array}{l}\text { Mahonia napaulensis DC. } \\
\text { NAH-142 }\end{array}$ & Berberidaceae & $\begin{array}{l}\text { Bhutro/ } \\
\text { Jamane } \\
\text { mandro }\end{array}$ & Shrub & Stem & Latex & Topical & Conjunctivitis & {$[30,40,51,59,63]$} \\
\hline 63. & $\begin{array}{l}\text { Malvaviscus arboreus Cav. } \\
\text { NAH- } 47\end{array}$ & Malvaceae & $\begin{array}{l}\text { Barhamase } \\
\text { ful/khursani } \\
\text { ful }\end{array}$ & Shrub & Root & $\begin{array}{l}\text { Juice and } \\
\text { decoction }\end{array}$ & Oral & Fever & None \\
\hline
\end{tabular}


Table 1. Cont.

\begin{tabular}{|c|c|c|c|c|c|c|c|c|c|}
\hline S.N. & $\begin{array}{l}\text { Botanical Name } \\
\text { Voucher Number }\end{array}$ & Family & Local Name & Habit & Parts Used & Preparation & $\begin{array}{l}\text { Mode of } \\
\text { Application }\end{array}$ & Ailments Treated/ Uses & $\begin{array}{l}\text { Previous Uses } \\
\text { Reported References }\end{array}$ \\
\hline \multirow[b]{2}{*}{64.} & \multirow[b]{2}{*}{$\begin{array}{l}\text { Mirabilis jalapa L. } \\
\text { NAH-49 }\end{array}$} & \multirow[b]{2}{*}{ Nyctaginaceae } & \multirow[b]{2}{*}{ Seto malati } & \multirow[b]{2}{*}{ Shrub } & Root & Paste & Topical & Inflammation & \multirow[b]{2}{*}{ [69] } \\
\hline & & & & & Rhizome & $\begin{array}{l}\text { Chewable, Juice } \\
\text { and decoction }\end{array}$ & Oral & $\begin{array}{l}\text { Diabetes mellitus, } \\
\text { Gastritis, Flatulence, } \\
\text { Body ache }\end{array}$ & \\
\hline 65. & $\begin{array}{l}\text { Mentha arvensis L. } \\
\text { NAH-85 }\end{array}$ & Lamiaceae & Babari & Herb & Flower & $\begin{array}{l}\text { Juice and } \\
\text { decoction }\end{array}$ & Oral & Thirst & {$[36,49,63,80]$} \\
\hline 66. & $\begin{array}{l}\text { Mentha spicata L. } \\
\text { NAH-86 }\end{array}$ & Lamiaceae & Pudina & Herb & Whole plant & $\begin{array}{l}\text { Juice, Paste and } \\
\text { pickle }\end{array}$ & Oral & $\begin{array}{l}\text { Diarrhea, Dysentery, } \\
\text { Urine retention, } \\
\text { Stomachache, indigestion }\end{array}$ & $\begin{array}{l}{[7,11,22,30,44,47,53,} \\
57,59,63,73,81]\end{array}$ \\
\hline 67. & $\begin{array}{l}\text { Momordica charantia L. } \\
\text { NAH-28 }\end{array}$ & Cucurbitaceae & Tite karela & Herb & Fruit & $\begin{array}{l}\text { Juice, decoction, } \\
\text { and boiled fruit }\end{array}$ & Oral & Hypertension & {$[11,52,66]$} \\
\hline 68. & $\begin{array}{l}\text { Morus alba L. } \\
\text { NAH-119 }\end{array}$ & Moraceae & Kiu kafal & Tree & Bark & Juice and paste & Topical & Toothache & {$[66,76,81]$} \\
\hline 69. & $\begin{array}{l}\text { Musa } \times \text { paradisiaca } \mathrm{L} \text {. } \\
\text { NAH-06 }\end{array}$ & Musaceae & Kera & $\begin{array}{l}\text { Stoloniferous } \\
\text { plant }\end{array}$ & Corm & $\begin{array}{l}\text { Juice and } \\
\text { decoction }\end{array}$ & Oral & Thirst, Fever & {$[11,31,53,80]$} \\
\hline 70. & $\begin{array}{l}\text { Myrica esculenta Buch.-Ham. ex } \\
\text { D. Don } \\
\text { NAH-18 }\end{array}$ & Myricaceae & Ban Kafal & Tree & Bark & $\begin{array}{l}\text { Chewable } \\
\text { (bark) }\end{array}$ & Oral & $\begin{array}{l}\text { Diabetes mellitus, } \\
\text { Toothache }\end{array}$ & {$[13,28,51,58,78,80]$} \\
\hline 71. & $\begin{array}{l}\text { Nepeta cataria L. } \\
\text { NAH-83 }\end{array}$ & Lamiaceae & Charpate & Herb & $\begin{array}{l}\text { Leaf, whole } \\
\text { plant }\end{array}$ & Juice & Topical & $\begin{array}{l}\text { Cuts and Wounds, } \\
\text { Bleeding }\end{array}$ & None \\
\hline 72. & $\begin{array}{l}\text { Nephrolepsis cordifolia (L.) C. } \\
\text { Presl } \\
\text { NAH-39 }\end{array}$ & Nephrolepidaceae & Pani amala & Fern & Tuber & Juice & Oral & $\begin{array}{l}\text { Jaundice, Diabetes } \\
\text { mellitus, Hematuria }\end{array}$ & {$[11,12,30,36,78]$} \\
\hline 73. & $\begin{array}{l}\text { Nicotiana tobacum L. } \\
\text { NAH-80 }\end{array}$ & Solanaceae & $\begin{array}{l}\text { Surti/Kacho } \\
\text { paat }\end{array}$ & Herb & Leaf & $\begin{array}{l}\text { Paste and } \\
\text { expressed juice }\end{array}$ & Topical & $\begin{array}{l}\text { Infected wounds, } \\
\text { Pediculosis }\end{array}$ & {$[49,71,78]$} \\
\hline 74. & $\begin{array}{l}\text { Ocimum tenuiflorum L. } \\
\text { NAH-102 }\end{array}$ & Lamiaceae & Tulsi & Shrub & $\begin{array}{l}\text { Leaf and } \\
\text { whole plant }\end{array}$ & $\begin{array}{l}\text { Decoction and } \\
\text { juice }\end{array}$ & Oral & $\begin{array}{l}\text { Heart failure, Flu, cardiac } \\
\text { stimulant }\end{array}$ & {$[49,52,53,63,78]$} \\
\hline \multirow{2}{*}{75.} & \multirow{2}{*}{$\begin{array}{l}\text { Oryza sativa L. } \\
\text { NAH- } 48\end{array}$} & \multirow{2}{*}{ Poaceae } & \multirow{2}{*}{ Dhan } & \multirow{2}{*}{ Grass } & \multirow{2}{*}{ Fruit } & Decoction & Oral & Inflammation & \multirow{2}{*}{ [7] } \\
\hline & & & & & & Roasted fruit & Oral & Cough & \\
\hline 76. & $\begin{array}{l}\text { Oxalis corniculata } \mathrm{L} . \\
\text { NAH- } 58\end{array}$ & Oxalidaceae & Chari amilo & Herb & Whole plant & $\begin{array}{l}\text { Decoction and } \\
\text { juice }\end{array}$ & Oral & $\begin{array}{l}\text { Flatulence, Gastritis, } \\
\text { Diarrhea, Dysentery, } \\
\text { Fever, Common cold, } \\
\text { Typhoid }\end{array}$ & $\begin{array}{l}{[11,36,44,45,48,49,57-} \\
59,63,64,71,80]\end{array}$ \\
\hline
\end{tabular}


Table 1. Cont.

\begin{tabular}{|c|c|c|c|c|c|c|c|c|c|}
\hline S.N. & $\begin{array}{l}\text { Botanical Name } \\
\text { Voucher Number }\end{array}$ & Family & Local Name & Habit & Parts Used & Preparation & $\begin{array}{l}\text { Mode of } \\
\text { Application }\end{array}$ & Ailments Treated/ Uses & $\begin{array}{l}\text { Previous Uses } \\
\text { Reported References }\end{array}$ \\
\hline 77. & $\begin{array}{l}\text { Periploca calophylla (Wight) Falc. } \\
\text { NAH-31 }\end{array}$ & Apocynaceae & Chautajor & Shrub & $\begin{array}{l}\text { Leaf and } \\
\text { stem }\end{array}$ & $\begin{array}{l}\text { Powder mixed } \\
\text { with cooked rice } \\
\text { flour in butter } \\
\text { (Puwa) }\end{array}$ & Oral & Body ache, Backache & None \\
\hline 78. & $\begin{array}{l}\text { Physalis peruviana } \mathrm{L} \text {. } \\
\text { NAH-13 }\end{array}$ & Solanaceae & Isamgol & Herb & Fruit & Decoction & Oral & Fever, Thirst & None \\
\hline 79. & $\begin{array}{l}\text { Pogostemon benghalensis } \\
\text { (Brum.f.) Kuntze } \\
\text { NAH-134 }\end{array}$ & Lamiaceae & Rudilo & Shrub & Leaf & Juice & Oral & Sore throat, Typhoid & {$[11,40,49,63,78]$} \\
\hline 80. & $\begin{array}{l}\text { Potentilla lineata Trev. } \\
\text { NAH-69 }\end{array}$ & Rosaceae & Bajradanti & Herb & $\begin{array}{l}\text { Leaf and } \\
\text { root }\end{array}$ & Juice & Topical & Toothache & [58] \\
\hline 81. & $\begin{array}{l}\text { Prunus persica (L.) Batsch } \\
\text { NAH-100 }\end{array}$ & Rosaceae & Aaru & Tree & $\begin{array}{l}\text { Bark and } \\
\text { leaf }\end{array}$ & Decoction & Oral & Typhoid, Sore throat & {$[11,49,58,65,66,70]$} \\
\hline 82. & $\begin{array}{l}\text { Psidium guajava L. } \\
\text { NAH-43 }\end{array}$ & Myrtaceae & Belauti/Aamba & Tree & $\begin{array}{l}\text { Bud and } \\
\text { bark }\end{array}$ & Juice & Oral & $\begin{array}{l}\text { Diarrhea, Dysentery, } \\
\text { Hypertension }\end{array}$ & $\begin{array}{l}{[7,11,31,39,45,49,58,} \\
59,63,66,78,80]\end{array}$ \\
\hline \multirow{2}{*}{83.} & \multirow{2}{*}{$\begin{array}{l}\text { Rhododendron arboreum Sm. } \\
\text { NAH-108 }\end{array}$} & \multirow{2}{*}{ Ericaceae } & \multirow{2}{*}{$\begin{array}{l}\text { Laali } \\
\text { Gurans }\end{array}$} & \multirow{2}{*}{ Tree } & \multirow{2}{*}{ Flower } & $\begin{array}{l}\text { Chewable(flower } \\
\text { petal) }\end{array}$ & Oral & Throat obstruction & \multirow{2}{*}{$\begin{array}{l}{[22,30,31,36,37,39,40} \\
42,47,51,59,63,65,73 \\
74,78,80]\end{array}$} \\
\hline & & & & & & Juice & Oral & $\begin{array}{l}\text { Diarrhea, Dysentery, Dry } \\
\text { cough due to allergy }\end{array}$ & \\
\hline \multirow{2}{*}{84.} & \multirow{2}{*}{$\begin{array}{l}\text { Rubus ellipticus Sm. } \\
\text { NAH-117 }\end{array}$} & \multirow{2}{*}{ Rosaceae } & \multirow{2}{*}{ Ainselu } & \multirow{2}{*}{ Shrub } & $\begin{array}{l}\text { Bud and } \\
\text { roots }\end{array}$ & Juice & Oral & Diabetes mellitus & \multirow{2}{*}{$\begin{array}{l}{[22,28,30,31,36,39,40,} \\
45,51,58,59,63,80]\end{array}$} \\
\hline & & & & & $\begin{array}{l}\text { Buds and } \\
\text { leaf }\end{array}$ & Juice & Topical & Cutsand Wounds & \\
\hline \multirow{4}{*}{85.} & \multirow{4}{*}{$\begin{array}{l}\text { Rumex nepalensis Spreng. } \\
\text { NAH-115 }\end{array}$} & \multirow{4}{*}{ Polygonacae } & \multirow{4}{*}{ Halhale } & \multirow{4}{*}{ Herb } & Whole plant & Juice and paste & Topical & $\begin{array}{l}\text { Dislocated bone, Fracture, } \\
\text { Sprain, Eye troubles }\end{array}$ & \multirow{4}{*}{$\begin{array}{l}{[22,30,36,41,42,45,46,} \\
51,53,56,58,60,63,64, \\
71,74,75,80]\end{array}$} \\
\hline & & & & & Leaf & $\begin{array}{l}\text { Decoction and } \\
\text { cooked leaves }\end{array}$ & Oral & Constipation & \\
\hline & & & & & Leaf & Paste & Topical & $\begin{array}{l}\text { Fungal infection, Skin } \\
\text { disorders }\end{array}$ & \\
\hline & & & & & Root & Juice & Oral & Diarrhea & \\
\hline 86. & $\begin{array}{l}\text { Saccharum officinarum L. } \\
\text { NAH-29 }\end{array}$ & Poaceae & Ukhu & Grass & Stem & Juice & Oral & Jaundice & [7] \\
\hline
\end{tabular}


Table 1. Cont.

\begin{tabular}{|c|c|c|c|c|c|c|c|c|c|}
\hline S.N. & $\begin{array}{l}\text { Botanical Name } \\
\text { Voucher Number }\end{array}$ & Family & Local Name & Habit & Parts Used & Preparation & $\begin{array}{l}\text { Mode of } \\
\text { Application }\end{array}$ & Ailments Treated/ Uses & $\begin{array}{l}\text { Previous Uses } \\
\text { Reported References }\end{array}$ \\
\hline 87. & $\begin{array}{l}\text { Saurauia fasciculata Wall. } \\
\text { NAH-42 }\end{array}$ & Actinidiaceae & Goban & Tree & Bark & Juice & Oral & $\begin{array}{l}\text { Thirst, Postpartum } \\
\text { hemorrhage, Fever }\end{array}$ & None \\
\hline 88. & $\begin{array}{l}\text { Schima wallichii Choisy } \\
\text { NAH-78 }\end{array}$ & Theaceae & Chilaune & Tree & Bark & Latex & Topical & $\begin{array}{l}\text { Bleeding, Cuts and } \\
\text { Wounds }\end{array}$ & {$[31,36,49,63,78,80]$} \\
\hline 89. & $\begin{array}{l}\text { Solanum annuum C.V. Morton } \\
\text { NAH-51 }\end{array}$ & Solanaceae & Khursani & Herb & Fruit & Fried in oil & Topical & Snakebite, Scabies & None \\
\hline \multirow{2}{*}{90.} & \multirow{2}{*}{$\begin{array}{l}\text { Solanum melongena L. } \\
\text { NAH-46 }\end{array}$} & \multirow{2}{*}{ Solanaceae } & \multirow{2}{*}{ Bhanta } & \multirow{2}{*}{ Herb } & Unripe fruit & Decoction & Oral & Fever & \multirow{2}{*}[7]{} \\
\hline & & & & & Bud & Paste & Topical & Burns, Boils & \\
\hline 91. & $\begin{array}{l}\text { Solanum pseudocapsicum L. } \\
\text { NAH-03 }\end{array}$ & Solanaceae & Tite bee & Shrub & Fruit & Juice & Topical & Headache, Toothache & None \\
\hline 92. & $\begin{array}{l}\text { Solanum torvum Sw. } \\
\text { NAH-95 }\end{array}$ & Solanaceae & $\begin{array}{l}\text { Kantakari } \\
\text { (Thulo Bihi) }\end{array}$ & Shrub & Fruit & Smoke & Inhalation & Toothache & None \\
\hline 93. & $\begin{array}{l}\text { Stephania japonica (Thunb.) } \\
\text { Miers } \\
\text { NAH-82 }\end{array}$ & Menispermacae & $\begin{array}{l}\text { Chillo } \\
\text { badulpate }\end{array}$ & Climber & Leaf & Decoction & Oral & Postpartum hemorrhage & [31] \\
\hline 94. & $\begin{array}{l}\text { Swertia chirayita L. } \\
\text { NAH-113 }\end{array}$ & Gentianaceae & Chiraaito & Herb & Leaf & $\begin{array}{l}\text { Juice and } \\
\text { decoction }\end{array}$ & Oral & $\begin{array}{l}\text { Fever, Headache, } \\
\text { Common cold, Gastritis, } \\
\text { Constipation }\end{array}$ & $\begin{array}{l}{[22,28,39-41,47,50,51,} \\
57,63,66,78]\end{array}$ \\
\hline 95. & $\begin{array}{l}\text { Tectaria coadunata (J. Sm.) C. } \\
\text { Chr. } \\
\text { NAH-41 }\end{array}$ & Dryopteridaceae & $\begin{array}{l}\text { Kalo } \\
\text { kuthurke }\end{array}$ & Fern & Root & Juice & Oral & Diarrhea, Dysentery & [28] \\
\hline \multirow{2}{*}{96.} & \multirow{2}{*}{$\begin{array}{l}\text { Thysanolaena latifolia (Roxb. ex } \\
\text { Hornem) Honda } \\
\text { NAH-21 }\end{array}$} & \multirow{2}{*}{ Poaceae } & \multirow{2}{*}{ Amriso } & \multirow{2}{*}{ Grass } & \multirow{2}{*}{ Root } & Paste & Topical & Breast engorgement & \multirow{2}{*}{ None } \\
\hline & & & & & & Decoction & Oral & Fever & \\
\hline 97. & $\begin{array}{l}\text { Trigonella foenum-graceum L. } \\
\text { NAH-143 }\end{array}$ & Fabaceae & Methi & Herb & Fruit & $\begin{array}{l}\text { Soup of roasted } \\
\text { fruit }\end{array}$ & Oral & Common cold, Cough & {$[22,39,52,56]$} \\
\hline 98. & $\begin{array}{l}\text { Urtica parviflora Roxb. } \\
\text { NAH-79 }\end{array}$ & Urticaceae & Sisno & Shrub & $\begin{array}{l}\text { Leaf and } \\
\text { Bud }\end{array}$ & $\begin{array}{l}\text { Juice and } \\
\text { decoction }\end{array}$ & Oral & $\begin{array}{l}\text { Blindness, Jaundice, } \\
\text { Urine retention }\end{array}$ & None \\
\hline 99. & $\begin{array}{l}\text { Viburnum mullaha Buch.-Ham. } \\
\text { ex D. Don } \\
\text { NAH-89 }\end{array}$ & Adoxaceae & Molo & Tree & Fruit & Juice & Oral & Poisoning & {$[30]$} \\
\hline
\end{tabular}


Table 1. Cont.

\begin{tabular}{|c|c|c|c|c|c|c|c|c|c|}
\hline S.N. & $\begin{array}{l}\text { Botanical Name } \\
\text { Voucher Number }\end{array}$ & Family & Local Name & Habit & Parts Used & Preparation & $\begin{array}{l}\text { Mode of } \\
\text { Application }\end{array}$ & Ailments Treated/ Uses & $\begin{array}{l}\text { Previous Uses } \\
\text { Reported References }\end{array}$ \\
\hline 100. & $\begin{array}{l}\text { Viola canescens Wall. } \\
\text { NAH- } 24\end{array}$ & Violaceae & $\begin{array}{l}\text { Aankhle } \\
\text { jhar }\end{array}$ & Herb & Leaf & Paste & Topical & Gout, Joint pain & {$[58,70]$} \\
\hline 101. & $\begin{array}{l}\text { Woodfordia fruticosa (L.) Kurz } \\
\text { NAH-17 }\end{array}$ & Lythraceae & Dhairo & Shrub & Flower & Juice & Oral & Diarrhea, Dysentery & $\begin{array}{l}{[11,44,47,49,57,59,63,} \\
70,78,79]\end{array}$ \\
\hline \multirow[b]{2}{*}{102.} & \multirow[b]{2}{*}{$\begin{array}{l}\text { Zanthoxylum armatum DC. } \\
\text { NAH-144 }\end{array}$} & \multirow[b]{2}{*}{ Rutaceae } & \multirow[b]{2}{*}{$\begin{array}{l}\text { Aakhe } \\
\text { Timur }\end{array}$} & \multirow[b]{2}{*}{ Shrub } & \multirow[b]{2}{*}{ Fruit } & Juice and paste & Topical & Snakebite, Scabies & \multirow[b]{2}{*}{$\begin{array}{l}{[13,30,31,36,37,40,42,} \\
46,47,50,51,57,59,60, \\
63,70,71,73,76,78,80]\end{array}$} \\
\hline & & & & & & $\begin{array}{l}\text { Juice and } \\
\text { decoction }\end{array}$ & Oral & $\begin{array}{l}\text { Gastritis, Intestinal } \\
\text { worms, Toothache, } \\
\text { Poisoning }\end{array}$ & \\
\hline 103. & $\begin{array}{l}\text { Zea mays L. } \\
\text { NAH-15 }\end{array}$ & Poaceae & Makai & Grass & Seed & Flour & Oral & Diabetes mellitus & {$[6,7,56,65,66]$} \\
\hline \multirow[b]{2}{*}{104.} & \multirow{2}{*}{$\begin{array}{l}\text { Zingiber officinale Roscoe } \\
\text { NAH-64 }\end{array}$} & \multirow[b]{2}{*}{ Zingiberaceae } & \multirow[b]{2}{*}{ Aduwa } & \multirow[b]{2}{*}{ Herb } & \multirow[b]{2}{*}{ Rhizome } & Paste & Topical & Snakebite & \multirow{2}{*}{$\begin{array}{l}{[6,11,22,49,50,52,58,} \\
73]\end{array}$} \\
\hline & & & & & & Decoction & Oral & $\begin{array}{l}\text { Vomiting, Stomachache, } \\
\text { Common cold }\end{array}$ & \\
\hline 105. & $\begin{array}{l}\text { Ziziphus mauritiana Lam. } \\
\text { NAH-44 }\end{array}$ & Rhamnaceae & Bayer & Tree & Seed & Powder & Chewing & Chickenpox, Measles & $\begin{array}{l}{[6,11,30,47,49,53,57,} \\
63,65,66]\end{array}$ \\
\hline
\end{tabular}




\subsection{Use of Plants for Primary Health Care}

It was found that local people used 105 medicinal plants to treat 70 different types of diseases and ailments (Table 1). These diseases and ailments were grouped into 13 different categories (Table 2) based on the International Classification of Primary Care (ICPC) i.e., digestive (37 species), skin (27 species), respiratory (19 species), musculoskeletal (12 species), circulatory (11 species), endocrine, metabolic, and nutritional (10 species), pregnancy, childbearing, family planning (5 species), eye (4 species), urinary (4 species), neurological (3 species), ear (2 species), female genital system (1 species), and general and unspecified (35 species). The information regarding the use of plant species for specific categories of diseases and ailments is listed in Table 2. Similarly, the biomedical and emic use reports are presented in Table 3.

Table 2. List of plant species used for specific categories of diseases and ailments.

\begin{tabular}{|c|c|c|}
\hline $\begin{array}{l}\text { Disease and Ailment } \\
\text { Categories }\end{array}$ & Name of Plants & No of Plants \\
\hline Musculoskeletal & $\begin{array}{l}\text { B. ciliata, B. alnoides, C. cristata, C. spinulosa, F. vulgare, H. lanceolata, } \\
\text { L. cubeba, M. jalapa, O. sativa, P. calophylla, R. nepalensis, V. canescens }\end{array}$ & 12 \\
\hline Digestive & $\begin{array}{l}\text { A. bidentata, A. calva, A. bicolor, A. sativum, B. purpurea, B. aristata, } \\
\text { C. asiatica, C. album, C. tamala, C. medica, C. aurantifolia, C. esculenta, } \\
\text { C. pepo, C. dactylon, D. carota, D. bipinnata, D. bulbophylla, D. diandra, } \\
\text { J. curcas, L. usitatissimum, L. cubeba, M. jalapa, M. spicata, } \\
\text { N. cordifolia, O. corniculata, P. guajava, R. arboreum, R. nepalensis, } \\
\text { S. officinarum, S. pseudocapsicum, S. torvum, S. chirayita, T. coadunata, } \\
\text { U. parviflora, W. fruticosa, Z. armatum, Z. officinale }\end{array}$ & 37 \\
\hline Eye & D. carota, M. napaulensis, R. nepalensis, U. parviflora & 4 \\
\hline Ear & B. pinnatum, M. arboreus & 2 \\
\hline Circulatory & $\begin{array}{l}\text { A. adenophora, A. sessilis, A. racemosus, C. verutum, C. limon, } \\
\text { E. prostrata, E. sonchifolia, M. charantia, N. cataria, O. tenuiflorum, } \\
\text { P guagava }\end{array}$ & 11 \\
\hline Neurological & C. arabica, S. pseudocapsicum, S. chirayita & 3 \\
\hline Respiratory & $\begin{array}{l}\text { A. bidentata, A. calamus, B. malabarica, C. asiatica, C. verutum, } \\
\text { C. longa, D. diandra, E. coracana, E. stricta, E. acuminata, J. curcas, } \\
\text { O. sativa, O. corniculata, P. benghalensis, P. persica, R. arboreum, } \\
\text { S. chirayita, T. foenum-graceum, Z. officinale }\end{array}$ & 19 \\
\hline Urinary System & A. bidentata, M. spicata, N. cordifolia, U. parviflora & 4 \\
\hline Skin & $\begin{array}{l}\text { A. adenophora, A. vera, A. sessilis, A. dubia, A. indica, C. aurantifolia, } \\
\text { D. falcatum, E. prostrata, E. sonchifolia, E. spicata, F. religiosa, G. hirta, } \\
\text { J. curcas, J. regia, L. cubeba, L. esculentum, L. ovalifolia, M. pustulata, } \\
\text { M. chisia, N. cataria, N. tobacum, R. ellipticus, R. nepalensis, } \\
\text { S. wallichii, S. annuum, S. melongena, Z. armatum }\end{array}$ & 27 \\
\hline $\begin{array}{l}\text { Endocrine, Metabolic } \\
\text { and Nutritional }\end{array}$ & $\begin{array}{l}\text { A. racemosus, C. verutum, C. aurantifolia, M. jalapa, M. esculenta, } \\
\text { N. cordifolia, P. peruviana, R. ellipticus, V. canescens, Z. mays }\end{array}$ & 10 \\
\hline $\begin{array}{l}\text { Pregnancy, Childbearing, } \\
\text { Family Planning }\end{array}$ & A. triplinervis, C. pareira, D. racemosus, S. fasciculata, S. japonica & 5 \\
\hline $\begin{array}{l}\text { Female Genital System } \\
\text { (including Breast) }\end{array}$ & T. latifola & 1 \\
\hline General and Unspecified & $\begin{array}{l}\text { A. manihot, A. bidentata, A. calamus, A. sativum, A. indica, } \\
\text { B. malabarica, B. aristata, B. alnoides, C. asiatica, C. tamala, C. verutum, } \\
\text { C. limon, C. arabica, C. esculenta, C. longa, E. stricta, E. acuminata, } \\
\text { I. cylindrica, L. usitatissimum, M. arboreus, M. x paradisiacal, } \\
\text { O. tenuiflorum, O. corniculata, P. peruviana, P. benghalensis, P. persica, } \\
\text { S. fasciculata, S. annuum, S. melongena, S. chirayita, T. latifola, } \\
\text { V. mullaha, Z. armatum, Z. officinale, Z. mauritiana }\end{array}$ & 35 \\
\hline
\end{tabular}


Table 3. Categories of diseases and ailments based on body system, along with their biomedical and emic use reports.

\begin{tabular}{|c|c|c|}
\hline Ailments Categories & Biomedical Terms & Local Terms (Emic Use Reports) \\
\hline \multirow{9}{*}{$\begin{array}{l}\text { General and } \\
\text { Unspecified }\end{array}$} & Chest pain & Chati dukheko \\
\hline & Chickenpox & Theula \\
\hline & Fever & Jworo aayeko \\
\hline & Flu & Bela Bela dekhaparne rughakoki, joro \\
\hline & Food poisoning & Khana ma bish pareko \\
\hline & Lethargy & Aalasya vayeko, alchi lageko \\
\hline & Measles & Dadura \\
\hline & Snakebite & Sarpa le tokeko \\
\hline & Typhoid & Kukhat pareko \\
\hline \multirow{17}{*}{ Digestive } & Anorexia & Khana ruchi nahune/ bhok nalagne/ aman hune \\
\hline & Constipation & Kabjiyet hune/ pet safa nahune/ disha garda garo hune \\
\hline & Diarrhea & Pakhala lageko/ cherpate chaleko/ pani jasto patalo disha aaune \\
\hline & Dysentery & Aaun pareko \\
\hline & Emesis & Banta garaune \\
\hline & Flatulence & Pet ma hawa variyeko/ bayu gola le pet dukhne \\
\hline & Gastric troubles & Pet ko gadbadi/ pet dhadiyeko \\
\hline & Gastritis & Gastric vayeko/mukh ma amilo pani aaune/ chati polne \\
\hline & Gingivitis & Gija sunniyeko \\
\hline & Indigestion & Apach/ khana apach hune \\
\hline & Intestinal worms infestation & Pet ma juka pareko \\
\hline & Jaundice & Pahele rog/jaundice vayeko \\
\hline & Nausea & Banta hola jasto hune/wakwak lagne \\
\hline & Stomachache & Pet dukheko \\
\hline & Throat obstruction & Ghanti ma adkeko, ghanti ma kei kura aljeko \\
\hline & Toothache & Dant dukheko/ dant kirale khayeko \\
\hline & Vomiting & Banta hune/ulti hune \\
\hline \multirow{4}{*}{ Eye } & Blindness & Aandho hune \\
\hline & Conjunctivitis & Aankha pakne rog \\
\hline & Corneal scar & Aankha ma phulo parne \\
\hline & Eye troubles & Aankha ka samasyaharu \\
\hline Ear & Earache & Kan dukheko \\
\hline \multirow{4}{*}{ Circulatory } & Bleeding & Dherai ragat bagne \\
\hline & Blood purification & Ragat safa garne \\
\hline & Heart failure & Mutu fail hune/ hridayaghat \\
\hline & Hypertension & Uchha raktachap, blood pressure badeko \\
\hline \multirow{8}{*}{ Musculoskeletal } & Backache & Dhad dhukeko/ kammar dukheko \\
\hline & Bodyache & Jyan dukheko \\
\hline & Bone weakness & Haddi ko kamjori \\
\hline & Dislocated bone & Haddi bhachiyera thau sareko \\
\hline & Fracture & Haddi futeko/bhachiyeko \\
\hline & Inflammation & Sunniyeko \\
\hline & Joint pain & Jorni ko dukhai \\
\hline & Sprain & Markeko \\
\hline Neurological & Headache & Tauko dukheko \\
\hline \multirow{8}{*}{ Respiratory } & Asthma & Dam vayeko/ sas ferna garo hune rog \\
\hline & Common cold & Rugha khoki lagne \\
\hline & Cough & Khoki lageko/ kaso lageko \\
\hline & Dry cough due to allergy & Allergy le garda hune sukka khoki \\
\hline & Nose bleeding & Naak bata ragat bagne \\
\hline & Sinusitis & Pinas bhayeko \\
\hline & Sore throat & Ghanti basne \\
\hline & Tonsillitis & Ghanti dukheko \\
\hline
\end{tabular}


Table 3. Cont.

\begin{tabular}{lll}
\hline Ailments Categories & Biomedical Terms & Local Terms (Emic Use Reports) \\
\hline & Boils & Pilo \\
& Burns & Poleko/ dadeko \\
& Cuts & Kateko \\
& Fungal infections & Sarir ma daj aaune \\
& Infected wounds & Ghau pakeko \\
Skin & Pediculosis & Sarirma jumra parne \\
& Pimple & Dandifor aayeko \\
& Scabies & Luto/ suke luto aayeko \\
& Skin disorders & Chala ko samasya/ chala ko rog/charma rog \\
& Tinea pedis/ ring worm & Kash ma daj aayeko \\
& Wounds & Ghau lageko \\
\hline \multirow{2}{*}{$\begin{array}{l}\text { Endocrine, Metabolic } \\
\text { and Nutritional }\end{array}$} & Diabetes mellitus & Chini rog, Madhumeha \\
& Gout & Bath rog \\
Thirst & Kharo hune \\
\hline Urinary System & Hematuria & Pisab ma ragat dekhine \\
& Urine retention & Pisab banda vayeko \\
\hline $\begin{array}{l}\text { Pregnancy, } \\
\text { Childbearing, Family }\end{array}$ & Post-partum hemorrhage & Sutkeri veyepachi dherai ragat bagne/ khanaro hune \\
\hline $\begin{array}{l}\text { Female Genital System } \\
\text { (including Breast) }\end{array}$ & Breast engorgement & Stan ma thunelo aayeko \\
\hline
\end{tabular}

\subsection{Plant Parts Used and Their Growth Forms}

Almost all parts of the plants were used to prepare medicine (leaf, root, rhizome, tuber, bulb, pseudobulb, corm, stem, bark, pith, flower, fruit, seed, bud, shoot, and whole plant) however, the most common plant parts used were underground parts including the root, rhizome, tuber, bulb, pseudobulb, and corm (used in 28 species), followed by fruit and seed ( 25 species), leaf ( 23 species), stem, bark, and pith (21 species), whole plant (17 species), bud and shoot (8 species), and flower (4 species). Additionally, the data of growth forms of plants indicate that most of the people used herbs $(40 \%)$, followed by trees $(24 \%)$, shrubs $(18 \%)$, grasses $(8 \%)$, climbers $(5 \%)$, ferns $(3 \%)$, orchids $(1 \%)$, and stoloniferous (1\%). Information regarding the number of plant species of each growth form is demonstrated in Figure 2. 


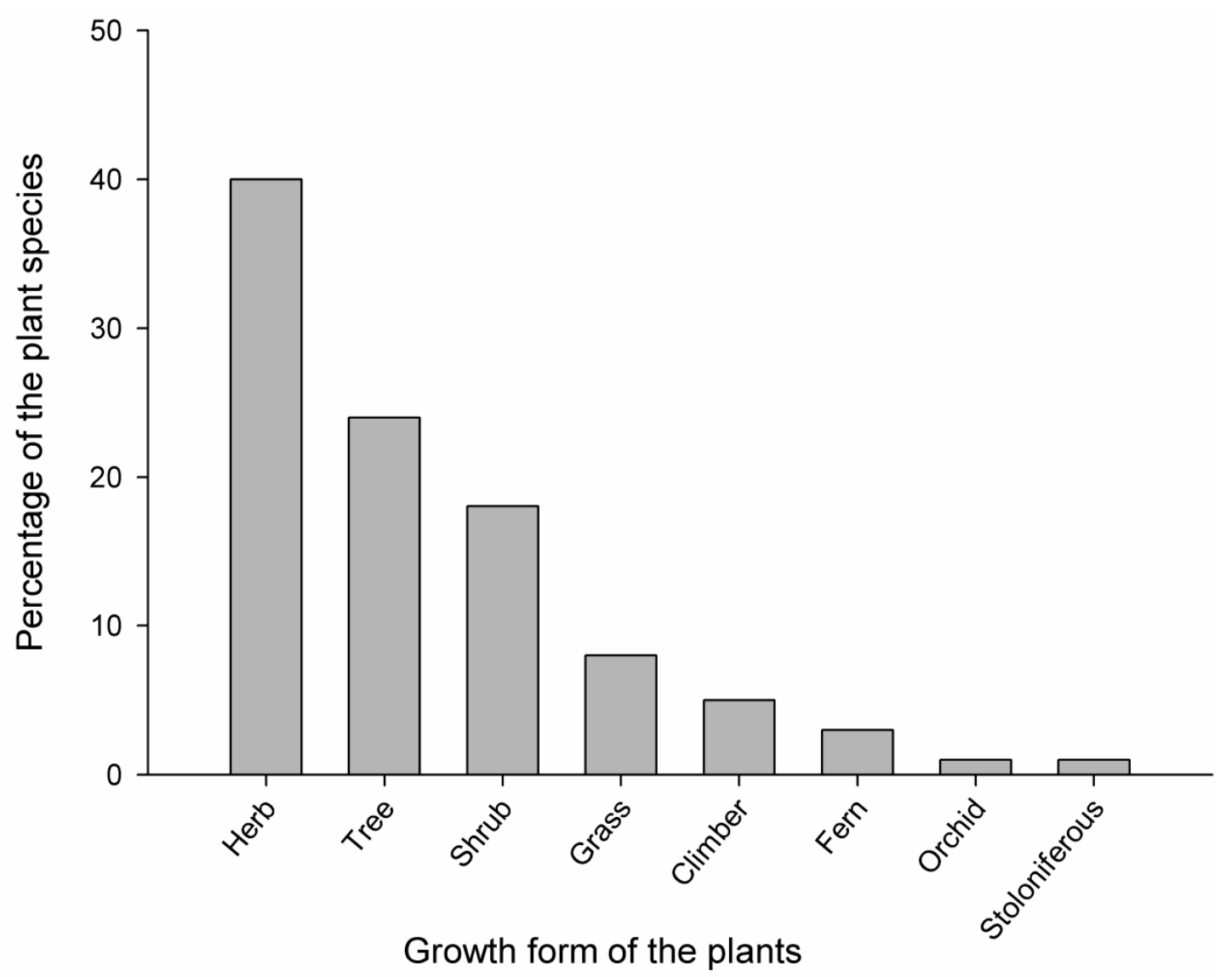

Figure 2. Growth form of the plant species used by the local people.

\subsection{Dosage Forms and Routes of Administration}

The study revealed that medicinal plants were mostly used in the decoction, juice, powder, paste, gel, latex, and pickled forms. Less frequently used formulations were roasted, vapor, boiled, and in traditional dishes like Puwa (plant powder cooked with rice flour in butter). Few plant species were also taken in chewable form. It was found that the most frequently used dosage form was juice (50 ailments), followed by paste (33 ailments), decoction (31 ailments), chewable (11 ailments), pickled (8 ailments), latex ( 7 ailments), gel ( 2 ailments), and other (17 ailments). Similarly, the oral route was found to be the most preferable route ( 77 plants) followed by the topical route (37 plants), the inhalation route ( 2 plants), and the aural route ( 2 plants). None of the plant preparations were taken through the parenteral route. The diagrammatic representation of the dosage form and their routes of administration are displayed in Figures 3 and 4, respectively. 


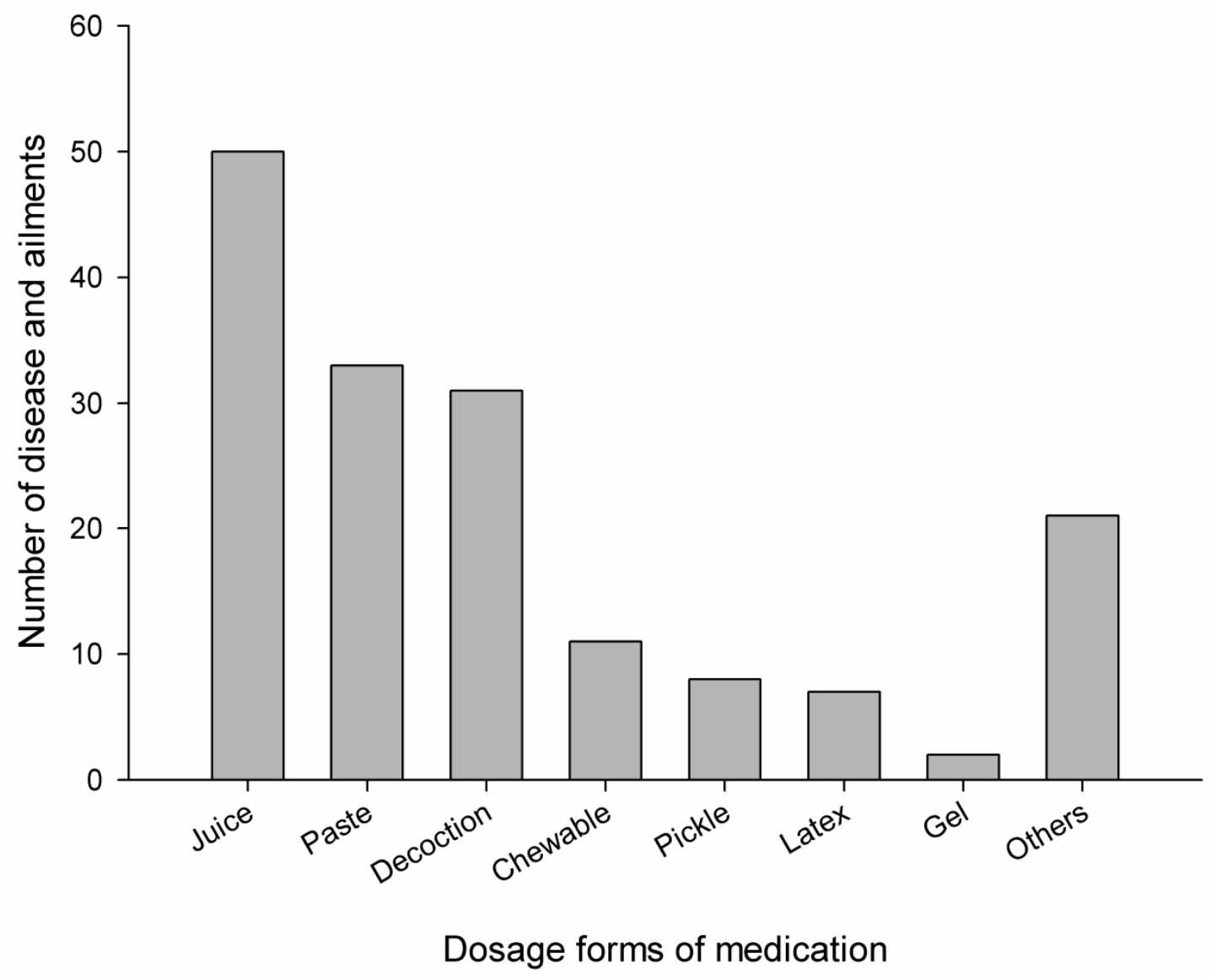

Figure 3. Dosage forms of medication in the treatment of different diseases and ailments by the local people.

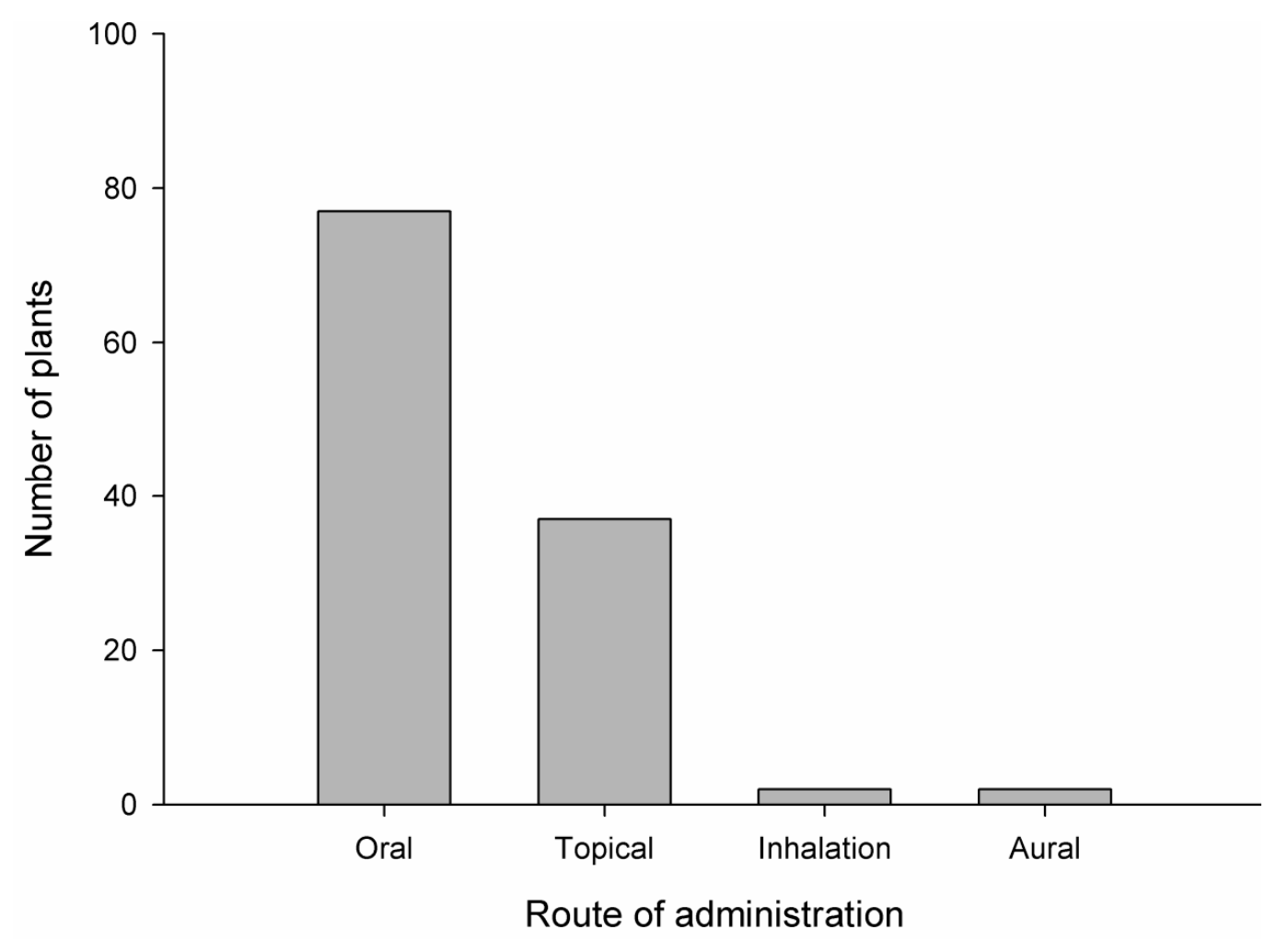

Figure 4. Routes of administration and the number of plants used by the local people. 


\section{Discussion}

\subsection{Plants and Usefulness}

Records of higher numbers of useful plant species from the plant families Poaceae, Asteraceae, and Fabaceae were already manifested in Nepal [82]. Poaceae, Asteraceae, and Fabaceae are the families with a large number of genera and species. It is often hypothesized that there exists a positive correlation between plant use/knowledge and plant density, diversity, and the habitat diversity [54,55]. If the area is biodiverse, the use of plants is heterogeneous and if the area is less diverse (abundant), the use of plants is homogenous [83]. The higher number of ethnomedicinal plants was found to be associated with the area rich in biodiversity.

It was found that the people of the Kaski district are rich in ethnomedicinal knowledge and mostly rely on plant-based remedies for common health problems like gastrointestinal disorders, respiratory disorders, musculoskeletal disorders, cardiovascular disorders, etc. The number of plants used to treat gastrointestinal disorders was found to be the highest (Figure 5), which is convergent to other studies considered when reviewing this study $[22,37,39,41,48,53]$. Most of the gastrointestinal problems were due to irregular dietary habit, poor hygiene, and contaminated food. Respiratory, diarrheal, and infectious diseases are also prevalent in Nepal and the country's sociological and topographical complexities burden health and sanitation [84]. Life in rural areas is further complicated [56] because of the effacement of traditional knowledge. Due to a lack of health education, people live with poor hygiene are vulnerable to many diseases. Benign health issues were frequently undermined because of a lack of health education. Some of the skin diseases like photo dermatitis [85], were caused due to prolonged exposure to sunlight during working hours. People suffered from fungal infections in the hands and feet, due to exposure to soil. Many people had musculoskeletal disorders due to greater physical stress, sprains, and fractures.

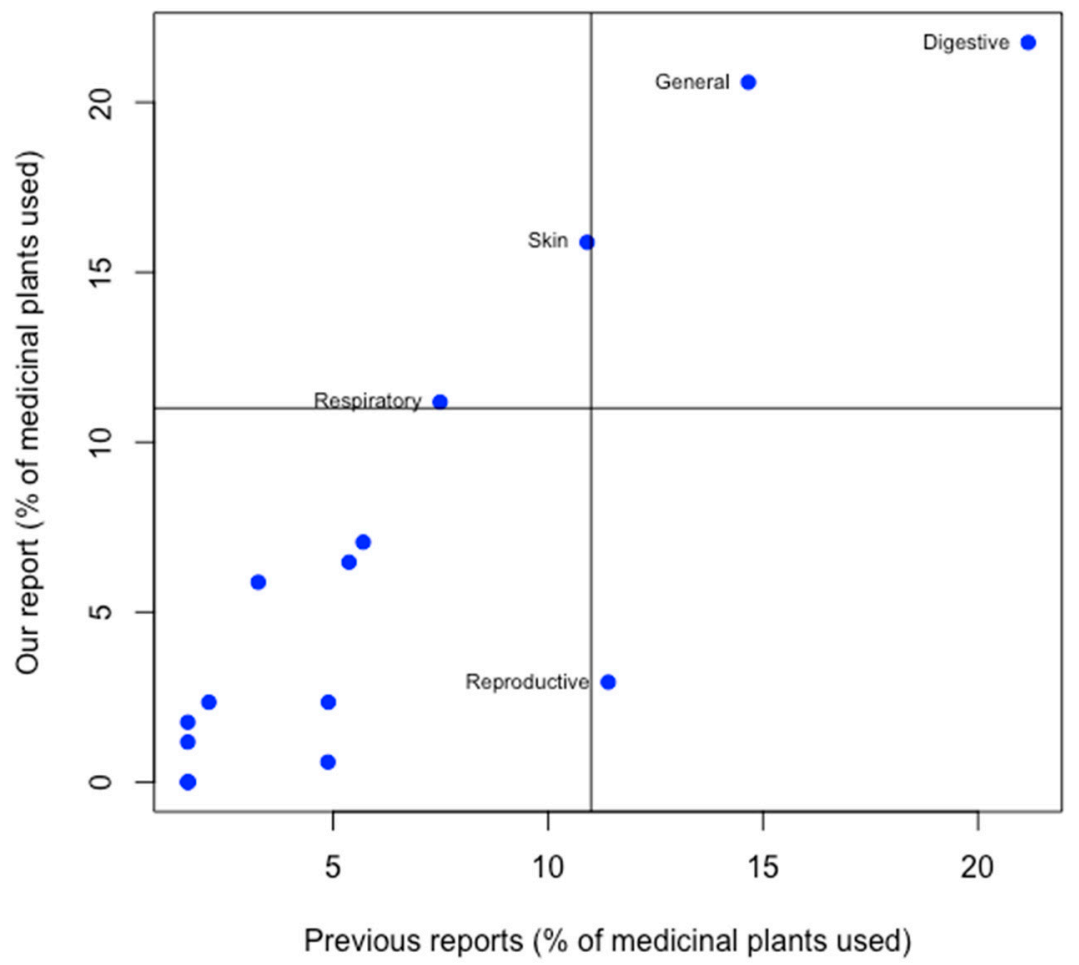

Figure 5. Comparison of the medicinal value of plants in the present study with previous studies in the Kaski district. 


\subsection{Perspective of People Towards Ethnomedicine}

We found that the elderly people and traditional healers were rich in ethnomedicinal knowledge and were more interested to conserve plants than younger people. Villagers believed in traditional healers, such as Dhami, Jhankri, and Pujari, as God's messengers and with super healing powers. They believe that plants become more medicinal when processed spiritually and materially. The traditional healers primarily used local medicinal plants to treat diseases. Besides local plants, they also used other important natural products available in other parts of the country. They generally exchanged their grains (such as rice, maize, etc.) with herbal ingredients brought by the transhumants from the Himalayan region.

Some of the elderly people claimed that they have not taken any allopathic medicine in their lifetime. However, they were less conscious of documentation and were unwilling to share their knowledge with others. They believed that if such secrets and their inherent knowledge is shared, the method does not work. They did not have any formal written documents about the use of medicinal plants. Most of the people in this area were not alarmed by the possible extinction of their indigenous knowledge. Few participants even told that traditional healers were remembered in their needs but not rewarded in return. Studies showed that younger people have little knowledge about plants and were inclined to allopathic medicines. However, they were interested in the documentation of their forefather's knowledge. Existing ethnomedicinal knowledge of these indigenous inhabitants has only been transmitted through the verbal prescription. This inherent and practical based knowledge is going to collapse soon, due to the declining interest of youngsters towards the value of traditional medicine and lack of specific plans and policies from the local government.

\subsection{Plant Parts Used and Their Growth Form}

Among the choice of plant parts, underground portions were found to be frequently used. Our finding bears a resemblance with much previous literature on the use of plant parts $[22,40,43,45$, $46,48,50,51,57-59]$. Regarding the preference of underground parts, the root was used in most of the cases, as it generally contains a greater amount of active principles, in comparison with other parts [60]. Like roots, leaves are also vulnerable parts of the plant and have a greater role in the plant defense system and possess higher concentrations of bioactive secondary metabolites $[49,61,86]$. This finding may be beneficial for choosing these parts for bioprospecting and bioactivity determination. However, improper and massive collections of roots may lead to complete destruction and decline of the plant from nature [62]. Herbs were found to be dominant growth form over others, being frequently used in Himalayan folk medicine and other countries $[7,13,22,30,39,40,43,48,50-53,56,58,59,63-66,86]$. The reason may be that our study site is located at a higher altitude, where trees are less abundant and other forms of plants are common [22]. It also may be due to the greater ease of collection, storage, transportation, and extraction of active compounds from herbs than other forms [51]. Herbs also contain higher amounts of secondary metabolites for their life strategies [67]. Herbs are relatively easy to cultivate, so they can easily fulfill the demand if needed in higher amounts [68]. Hence, our finding highlighted that the selection and use of herbs depend on factors like their phytochemical profile, their natural habitat, and their accessibility, partially supporting ecological apparency hypothesis.

\subsection{Dosage Forms and Route of Administration}

In this area, the juice was used most frequently, followed by paste, decoction, chewable, pickled, latex, and gel. This information resembles the data published by other studies carried out in Nepal and other countries $[7,51,53,59]$. The preference of juice may be due to the ease of preparation and its effectiveness [58]. It may also be due to the presence of a greater amount of active principles extracted in juice than other dosage forms [66]. It was also found that most of the herbal plants were used with hot water. Some plants were used along with milk, ghee, and honey. For example, flour of $O$. sativa 
and powder of $B$. ciliata were prepared in a ghee base, both for oral and local application, for backache and inflammation.

Most of the dosage forms were freshly prepared when needed. For example, a fresh paste of G. hirta leaves and latex of M. pustulata was applied around boils for faster healing purposes. A single plant can be used to treat many disorders. Examples of such plants included A. bidentata, A. sativum, A. sessilis, B. ciliata, C. verutum, C. esculenta, D. diandra, J. curcas, L. cubeba, M. arboreus, M. jalapa, O. tenuiflorum, O. sativa, R. arboreum, R. ellipticus, R. nepalensis, S. melongena, T. latifolia, Z. armatum, and $Z$. officinale. Plants were also used in combined forms. People said that paste and juice of $C$. asiatica and C. dactylon are used together for typhoid. Similarly, people confirmed that the paste of three plants, A. sativum, Z. armatum, and Z. officinale, is useful for treating snakebites by the expulsion of venom from the snakebite. The increased effect of the plants used together may be due to their synergistic effect. This increased bioactivity of the combined plants opens the door for the identification of compounds with similar mechanism of action. While comparing the choice of routes of drug administration, the oral route was found to be the most preferable route in this study area. The data from other studies also showed that people favor the oral route for medications $[22,41,48,51,56,59,61,64]$.

\subsection{Comparison of the Reported Uses}

We found that there are 47 plant species that have similar uses to treat 42 different ailments. A. bidentata was used in typhoid, tonsillitis, and toothache in this area. However, the same plant was found to be useful in the treatment of urinary disorder and indigestion in the Kavrepalanchok district [7] and asthma in Macchegaun, Kathmandu [36]. Another plant, A. calva, was used for dermatological disorders in the Rupandehi district of western Nepal [69]. However, this plant is used for gastrointestinal disorders like an intestinal worm, gastritis, and flatulence in the Kaski district. The rhizome of A. calamus was used in respiratory disorders like cough, chest pain, and asthma in our study area, which was also practiced in different regions of Nepal, like Rasuwa [13], Rupandehi [44,53], Humla [22], Macchegaun [36], Makawanpur [40], Gulmi [63], Dolpa [46], Nawalparasi [49], and Ghandruk [31]. The bulb of A. sativum fried in mustard oil was applied to treat earaches in Pakistan [70]. Similarly, its oil and fresh bulbs are used to cure skin rashes and blood pressure in the Kavrepalanchok district [7]. The decoction of this plant is taken for gastritis, flatulence, diarrhea, and dysentery in our study area and the paste of bulb is applied topically for snake bites. We found that people used the juice of A. vera for burns and boils. However, the same plant is used for diabetes in the Panchase village of the Kaski district [28], fever and cough in the Makawanpur district of Nepal [40], menstruation cramps, intestinal worms, and constipation in the Badgaun village of the Gulmi district [63], and rheumatism, constipation, and backache in Pakistan [56]. The importance of this plant for burns and boils in our area coincides with the accounts reported in Panchase, Kaski [28], Machhegaun, Kathmandu [36], and the Rudraprayag district of neighboring country India [66].

The inhabitants of Dekhatbuli of Kanchanpur districts used a paste of $A$. sessilis to treat wounds, venereal diseases, menstrual disorder, fever, and bloody dysentery [11]. The same plant has been reported for scabies in Rupandehi, Nepal [69] and bleeding, cuts, and wounds in Machhapuchchhre, Kaski, and Gulmi, Nepal [63]. Few previous studies showed leaves of B. pinnatum are beneficial for wounds, boils, skin burn, and are also applied to remove pus from the ear and vagina in Rupandehi, Nepal [69] and the plant is used to relieve ear pain in the Kaski district. Local people have been using the apical bud of C. verutum to treat sore throat and epistaxis like in Jajarkot district of Nepal [42,71]. Also this plant is used to treat urinary troubles in our study area which has same application in Karnali and Badagaun of the Gulmi district [63,71]. The practice of using the juice of the root of this plant for typhoid fever and diabetes is unique in our study. In the Mankawanpur district, the whole plant of D. bipinnata was used to correct thirst, fever, libido, asthma, and jaundice [40]. However, the paste of this plant is useful for tooth aches in our study area, which is justified by one study performed in Badagaun of the Gulmi district [63]. 
The root of $A$. bidentata contains phytosterols like sitosterol and sigma sterol, which have antibacterial activity, useful for toothaches [87]. The usefulness of this plant for typhoid and tonsillitis is also supported by the antibacterial activity of its ethanolic extract [88]. The plant $A$. calva is used for anthelmintic activity. This activity is justified by the lethality property of this extract against malarial and filarial vector [89]. A study showed that $A$. calamus possesses compounds like papaverine, with a dual inhibitor of the calcium channel and phosphodiesterase in the hexane fraction, and the anticholinergic compound rolipram, which has a phosphodiesterase- 4 inhibitor in the ethyl acetate fraction [90]. In addition, one study showed that the anti-asthmatic activity of this plant is devoid of side effects [91]. A decoction of A. sativum is indigenously taken for gastrointestinal disorders like flatulence, diarrhea, dysentery, and gastritis in our study area. This medicinal property is justified by its reported anti H. pylori activity [92-94]. During the survey, we encountered the plant $A$. sessilis, which is beneficial for bleeding, cuts, and wounds. These properties can be verified by one study, where a chloroform extract of the leaves showed significant wound healing activity [95]. Though the antipyretic activity of this plant is not yet reported, our research findings will open a door for screening for its antipyretic property. The leaves extract of B. purpurea contain flavonoids and tannins, which exhibit antiulcer activity in rats. This result provides the effectiveness of this plant for the prevention and treatments of gastric ulcers [96]. Additionally, the antimicrobial protein isolated from the seeds of this plant justifies its beneficial effects in gastritis [97]. Folk use of stem and root juice of B. aristata for typhoid fever, jaundice, and diarrhea is supported by its reported antibacterial activity against Salmonella typhi and its hepatoprotective and anti-diarrheal activity, respectively [98-103]. One in vivo experiment showed that the same plant contains an excess amount of berberine, an alkaloid responsible for hepatoprotective effects by lowering hepatotoxicity, oxidative and nitrosamine stress, TNF- $\alpha$, and iNOS levels. The inflammatory modulatory effect of this plant in the liver favors its effectiveness in the treatment of jaundice [104]. Experiments performed by Somania et al. [105] showed that an ethanolic extract of $A$. racemosus reduced blood glucose level in streptozotocin-induced diabetic rats. Hence, the reported use of this plant in the treatment of diabetes is justified.

C. asiatica is indigenously used to cure typhoid fever, cough, tonsillitis, and gastritis in this study area. The purport of indigenous uses of this plant was substantiated by its pharmacological activities (antibacterial, antiulcer, anti-allergic, anti-spasmodic, antitussive, antiviral, and anti-inflammatory activity) shown by compounds like apigenin, luteolin, $\alpha$-pinene, and $\beta$-pinene [106]. Furthermore, the beneficial effect of C. asiatica is justified by its anti-ulcer activity shown in animal models [107]. The reported osteoporotic activity of the ethanolic extract and isolated compound coelogin from C. cristata justify its traditional use for backache, fractures, and sprains [108]. Its traditional dish, called Puwa, is made from the powder of its pseudobulb and is found to be very popular among the villagers for these musculoskeletal disorders. D. carota is indigenously used to treat jaundice and blindness in this study area. This indigenous use is proved by its antioxidant, hepatoprotective, and intraocular pressure reducing effect [109-112]. Similarly, F. vulgare has greater uses for musculoskeletal problems, like fractures and bone weakness. This medicinal potency is also validated by its osteogenesis inducing effect in human mesenchymal cells and its inhibitory effect on osteoclast differentiation and ovariectomy induced bone loss $[113,114]$. Traditional use of $J$. curcas in gingivitis, tonsillitis, and sore throat is proved by its positive in vitro antibacterial property against various susceptible bacteria $[115,116]$. The usefulness of $J$. regia for fungal infection is scientifically confirmed by its fungistatic effect in previous studies [117]. Trigonelline, a potent alkaloid found in $M$. jalapa, has a $\beta$ cell protective effect with a good antioxidative potential [118]. This finding supports the clinical use of this plant for diabetes. Additionally, the effectiveness of this plant in the prevention and treatment of diabetes is supported by another hypoglycemic study performed by Zhou et al. [119] in streptozotocin-induced diabetic rats. Experiments performed by Sasa et al. [120] showed that the hypotensive effect of M. charantia is due to momordin, a saponin present in this plant. This compound has the potential to activate the peroxisome-proliferator activated-receptor (PPAR) delta gene in humans. The activation of this 
gene is responsible for the formation of high-density lipoproteins (HDL), which leads to a decrease in cardiovascular disorders like atherosclerosis and associated hypertension.

In our study area, $M$. alba is extensively used to reduce toothache. This anti-caries activity is proved by two experiments performed by Lokegaonkar et al. [121] and Islam et al. [122], wherein they found that both the plant extract and the isolated compound 1-deoxynojirimycin worked against Streptococcus mutans, a bacterium responsible for causing dental plaque and tooth decay. Similarly, the antibacterial property of this plant against oral pathogens is supported by the isolation of potent antibacterial flavonoids like cyclocommunol $(\mathrm{Cy}, 1)$, morusin $(\mathrm{Mi}, 3)$, kuwanon $\mathrm{G}$, and kuwanon $\mathrm{E}(\mathrm{Ku}, 4)[123,124]$. Hence, the ethnomedicinal use of this plant for toothaches is also pharmacologically validated.

In the Kaski district, O. corniculata is used to correct gastritis. This beneficial effect is scientifically proved by its gastroprotective effect on experimentally induced gastric ulceration in Wistar rats [125]. P. guajava has been used to reduce blood pressure in our study area, which is justified by its bioactive compounds like guiajaverin and quercetin having anti-hypertensive property [126]. The ethnomedicinal value of R. ellipticus juice for cuts and wounds in many parts of our study area is favored by its wound healing effect in animal experiments [127]. We found that a decoction and cooked leaves of R. nepalensis is used for constipation and its paste is utilized for fungal infections. Literature also showed that the methanolic root extract of this plant has a purgative action in rats and also possesses antifungal activities $[125,128]$. S. officinarum juice contains polyphenolic flavonoids and possesses good antioxidant activity [129]. In addition, this plant is reported to have a hepatoprotective effect, which justifies its ethnomedicinal values for jaundice [130]. Similarly, the ethnomedicinal use of $U$. parviflora for jaundice is proved by its hepatoprotective potential in carbon tetrachloride challenged experimental rats [131]. W. fruticosa contains some antimicrobial terpenes like 2-methoxy-4-(2-propenyl) phenol, 2,6-octadien-1-ol,3,7-dimethyl-(E)-2,6-octadienal, 5-methyl-2-(1-methylethyl) phenol, 3,7 dimethylcyclohexanol, cyclohexanol, and 2-methylene-5-(1-methylethenyl), which may be responsible for the local remedies of this plant for diarrhea and dysentery [132]. An aqueous extract of the Z. armatum seed was reported to be active against worm infestation caused by Haemonchus contortus, which validates its application for intestinal worm in our study area [133]. Locally, flour of Z. mays is used to reduce blood glucose level. Previous studies proved that phenolic compounds isolated from Z. mays are effective in reducing complications associated with diabetes mellitus. Hence, the antidiabetic property of this plant in our study area is validated [134]. Z. officinale contains gingerol as an active ingredient, which possesses cholinergic $\mathrm{M}_{3}$ and $5-\mathrm{HT}_{3}$ receptor blocking effects, thereby decreasing gastric emptying time and leading to the prevention of nausea and vomiting [135]. The presence of gingerol, an antiemetic compound, justifies the traditional use of this plant to for the relief of nausea and vomiting.

From the above comparisons of ethnomedicinal uses of medicinal plants with their previous reported uses, it is found that there is a strong variation in their uses within Nepal and other parts of the world. Additionally, the medicinal uses of some plants were newly discovered in our study area and were not encountered in other studies. Such heterogeneity of ethnomedicinal uses of these plants may be due to the predominance in the geographical location and the difference in vegetation. In addition, we found that some of the plants included in our study were already reported for their biological activities and phytochemical constituents. These reported activities and phytochemical constituents are similar to the claimed medicinal uses in this region, which reliability justifies the reported uses. Thus, the ethnomedicinal plant species, which are scientifically validated, are recommended for further studies for advanced pharmacological screening and drug development.

\subsection{The Novelty of the Work and Future Prospects}

Acharya and Acharya [27] recorded 18 medicinal plants, belonging to 17 families, for treating livestock diseases in Machhapuchchhre (formerly Sardikhola village), in the Kaski district. Similarly, another study, conducted by Bhattarai et al. [28] in Panchase, Kaski, reported 45 plant species belonging to 32 families that treat 34 different ailments. Ethnobotanical study conducted by Adhikari and 
Fisher [31] reported 54 local plant species from the Ghandruk village located inside ACA. Kunwar [30] also reported 66 plant species from Bhadaure Tamagi of the Kaski district. Among all these reported medicinal plants in this study area, 81 of them were reported in the book "Plants and People of Nepal" [136]. Surprisingly, from this survey we were able to document 83 plant species being used to treat 53 different diseases and ailments for the first time. Among them, 22 medicinal plants appeared to have new ethnomedicinal values. These plants include A. adenophora, A. bicolor, B. malabarica, C. cristata, D. macrocapnos, D. racemosus, D. belophylla, E. acuminata, G. hirta, H. lanceolata, M. pustulata, M. chisia, M. arboreus, N. cataria, P. calophylla, P. peruviana, S. fasciculata, S. annuum, S. pseudocapsicum, S. torvum, T. latifolia, and U. parviflora. Thus, we can claim that Machhapuchchhre Rural Municipality of the Kaski district is highly rich in medicinal plant resources. This unexplored ethnomedicinal knowledge pool of this area should be well documented, validated, and promoted for the socioeconomic and health benefits of the local people. Phytochemial profile and biological activities of newly encountered plants are yet unknown. This opens the door for researchers to perform phytochemical and pharmacological studies for the discovery of novel bioactive constituents.

\section{Conclusions}

The present study revealed that the Machhapuchchhre Rural Municipality of the Kaski district in Nepal is rich in plant resources that are traditionally used as medicines. The people of this area have been using a variety of plants for treating different diseases and ailments. They have abundant indigenous knowledge about plant collection, dosage form preparation, and their utilization. A total of 105 medicinal plants, belonging to 58 families and 99 genera, which treat 70 different diseases and ailments, were documented. The medicinal values of 22 different plant species were recorded for the first time in the district. The medicinal properties of the plants were justified by comparing them with relevant literature published from different parts of the world. The positive correlation between the traditional uses of the reported plants and their experimentally proven pharmacological activities was established.

To recapitulate, this study provides authentic data regarding the ethnomedicinal uses of the local flora of this rural region and will draw the attention of pharmacognosists, pharmacologists, phytochemists, and traditional healers for conducting further research to find therapeutically active natural products. We recommend that the claimed medicinal plants of this area should be protected using appropriate conservation measures and that a scientific assessment of the plant-lore in the district is urgently needed.

Author Contributions: M.A. carried out field survey, collected ethnomedicinal data, processed voucher specimen, and wrote first draft of manuscript. R.T. carried out field survey, analyzed data, and contributed in manuscript writing. H.P.D. and R.M.K. provided the technical support during the manuscript writing and guided the ethnobotanical and ethnomedicinal prospects of the research findings. P.P. designed whole study, supervised the project work, and finalized the manuscript and write up processes. All authors read and agreed for submission of this manuscript.

Funding: This research received no external funding.

Acknowledgments: Authors are very grateful to local people of Machhapuchchhre Rural Municipality, Kaski district, Nepal for their active participation and sharing their knowledge during field survey. We are also thankful to National Herbarium and Plant Laboratories, Lalitpur for the identification of plant specimens and Annapurna Conservation Area Project (ACAP), headquarter, Pokhara for giving the permission to conduct research in Annapurna Conservation Area.

Conflicts of Interest: The authors declare no conflict of interest.

\section{References}

1. Thorsen, R.S.; Pouliot, M. Traditional medicine for the rich and knowledgeable: Ahallenging assumptions about treatment-seeking behaviour in rural and peri-urban Nepal. Health Policy Plan. 2016, 31, 314-324. [CrossRef] [PubMed]

2. WHO. WHO Traditional Medicine Strategy 2014-2023; World Health Organization: Geneva, Switzerland, 2013. 
3. Koirala, R.R.; Khaniya, B.N. Present Status of Traditional Medicines and Medicinal E Aromatic Plants Related Resources and Organizations in Nepal; Nepal Health Research Council: Kathmandu, Nepal, 2009.

4. Abbott, R. Documenting Traditional Medical Knowledge; World Intellectual Property Organization: Geneva, Switzerland, 2014.

5. Harvey, A.L.; Edrada-Ebel, R.; Quinn, R.J. The re-emergence of natural products for drug discovery in the genomics era. Nat. Rev. Drug Discov. 2015, 14, 111-129. [CrossRef] [PubMed]

6. Jayaprasad, B.; Thamayandhi, D.; Sharavanan, P.S. Traditionally using antidiabetic medicinal plants in Tamil Nadu. Int. J. Res. Pharm. Biosci. 2011, 2,1-8.

7. Malla, B.; Chhetri, R.B. Indigenous knowledge on ethnobotanical plants of Kavrepalanchowk district. Kathmandu Univ. J. Sci. Eng. Technol. 2009, 5, 96-109.

8. Cunningham, A.B.; Shanley, P.; Laird, S. Health, habitats and medicinal plant use. In Human Health and Forests: A Global Overview of Issues, Practice and Policy; Colfer, C.J.P., Ed.; Earthscan: London, UK, 2008; pp. 35-62.

9. Chhetri, H.P.; Yogol, N.S.; Sherchan, J.; KC, A.; Mansoor, S.; Thapa, P. Phytochemical and antimicrobial evaluations of some medicinal plants of Nepal. Kathmandu Univ. J. Sci. Eng. Technol. 2008, 1, 49-54. [CrossRef]

10. Dias, D.A.; Urban, S.; Roessner, U. A historical overview of natural products in drug discovery. Metabolites 2012, 2, 303-336. [CrossRef]

11. Dhami, N. Ethnomedicinal uses of plants in western Terai of Nepal: A case study of Dekhatbhuli VDC of Kanchanpur district. In Medicinal Plants in Nepal: An Anthology of Contemporary Research; Jha, P.K., Karmacharya, S.B., Chettri, M.K., Thapa, C.B., Shrestha, B.B., Eds.; Ecological Society (ECOS): Kathmandu, Nepal, 2008; pp. 164-176.

12. Sathiyaraj, G.; Muthukumar, T.; Ravindran, K.C. Ethnomedicinal importance of fern and fern allies traditionally used by tribal people of Palani Hills (Kodaikanal), Western Ghats, South India. J. Med. Herbs Ethnomed. 2015, 1, 4-9. [CrossRef]

13. Uprety, Y.; Asselin, H.; Boon, E.K.; Yadav, S.; Shrestha, K.K. Indigenous use and bio-efficacy of medicinal plants in the Rasuwa district, central Nepal. J. Ethnobiol. Ethnomed. 2010, 6, 3. [CrossRef]

14. UNEP. Green Economy Sectorial Study: Biotrade-Harnessing the Potential for Transitioning to a Green Economy-The Case of Medicinal and Aromatic Plants in Nepal; United Nations Environment Programme: Nairobi, Kenya, 2012.

15. CBS. Population Profile of Nepal; Central Bureau of Statistics: Kathmandu, Nepal, 2007.

16. Ignacimuthu, S.; Ayyanar, M.; Sivaraman K, S. Ethnobotanical investigations among tribes in Madurai district of Tamil Nadu (India). J. Ethnobiol. Ethnomed. 2006, 2, 25. [CrossRef]

17. Quave, C.; Pieroni, A. A reservoir of ethnobotanical knowledge informs resilient food security and health strategies in the Balkans. Nat. Plants 2015, 1, 14021. [CrossRef]

18. Gewali, M.B. Aspects of Traditional Medicine in Nepal; University of Toyama: Toyama, Japan, 2008.

19. Williams, N. On the trail of Nepal's flora. Curr. Biol. 2005, 15, R977-R978. [CrossRef]

20. FAO. Impact of cultivation and gathering of medicinal plants on biodiversity: Global trends and issues. Available online: http://www.fao.org/3/aa010e/AA010e00.htm (accessed on 23 June 2019).

21. Kunwar, R.M.; Mahat, L.; Acharya, R.P.; Bussmann, R.W. Medicinal plants, traditional medicine, markets and management in far-west Nepal. J. Ethnobiol. Ethnomed. 2013, 9, 24. [CrossRef] [PubMed]

22. Rokaya, M.B.; Munzbergova, Z.; Timsina, B. Ethnobotanical study of medicinal plants from the Humla district of western Nepal. J. Ethnopharmacol. 2010, 130, 485-504. [CrossRef] [PubMed]

23. MoFSC. Nepal Fifth National Report to Convention on Biological Diversity; Government of Nepal, Ministry of Forests and Soil Conservation: Kathmandu, Nepal, 2014.

24. Fransworth, N.R.; Morris, R.W. Higher plants - the sleeping gian of drug development. Am. J. Pharm. Educ. 1976, 148, 46-52.

25. Byg, A.; Salick, J.; Law, W. Medicinal plant knowledge among lay people in five eastern Tibet villages. Hum. Ecol. 2010, 38, 177-191. [CrossRef]

26. Kunwar, R.M.; Baral, K.; Paudel, P.; Acharya, R.P.; Thapa-Magar, K.B.; Cameron, M.; Bussmann, R.W. Land-use and socioeconomic change, medicinal plant selection and biodiversity resilience in far western Nepal. PLoS ONE 2016, 11. [CrossRef] [PubMed]

27. Acharya, K.P.; Acharya, M. Traditional knowledge on medicinal plants used for the treatment of livestock diseases in Sardikhola VDC, Kaski, Nepal. J. Med. Plant Res. 2010, 4, 235-239. 
28. Bhattarai, K.R.; Maren, I.E.; Chaudhary, R.P. Medicinal plant knowledge of the Panchase region in the middle hills of the Nepalese Himalayas. Banko Janakari 2011, 21, 31-39. [CrossRef]

29. Rana, S.K.; Oli, P.S.; Rana, H.K. Traditional botanical knowledge (TBK) on the use of medicinal plants in Sikles area, Nepal. Asian J. Plant Sci. Res. 2015, 5, 8-15.

30. Kunwar, B.B. Plants struggling to receive proper identity at Bhadaure Tamagi of Kaski district, Nepal. Trop. Plant Res. 2017, 4, 286-296. [CrossRef]

31. Adhikari, Y.P.; Fischer, A. Trend analysis and purpose of use of some important plant and animal species of Ghandruk VDC, Nepal. Our. Nat. 2010, 8, 122-130. [CrossRef]

32. CBS. Rural Municipality/Municipality's Profile of Kaski; Central Bureau of Statistics: Pokhara, Kaski; 2074 BS.

33. Bhuju, U.R.; Shakya, P.R.; Basnet, T.B.; Shrestha, S. Nepal Biodiversity Resource Book-Protected Areas, Ramsar Sites, and World Heritage Sites; ICIMOD: Lalitpur, Nepal, 2007.

34. Khadka, C.B.; Balla, M.K.; Tiwari, K.R. Assessment of climate change and its impact on cash crop in Lwang Ghalel of Kaski district. Banko Janakari 2011, 21, 31-34. [CrossRef]

35. Martin, G.J. Ethnobotany: A Methods Manual; Chapman \& Hall: London, UK, 1995.

36. Joshi, K.; Joshi, R.; Joshi, A. Indigenous knowldege and uses of medicinal plants in Macchegaun, Nepal. Indian J. Tradit. Knowl. 2011, 10, 281-286.

37. Kunwar, R.M.; Nepal, B.K.; Kshhetri, H.B.; Rai, S.K.; Bussmann, R.W. Ethnomedicine in Himalaya: A case study from Dolpa, Humla, Jumla and Mustang districts of Nepal. J. Ethnobiol. Ethnomed. 2006, 2, 27. [CrossRef] [PubMed]

38. Kunwar, R.M.; Shrestha, K.P.; Bussmann, R.W. Traditional herbal medicine in far-west Nepal: A pharmacological appraisal. J. Ethnobiol. Ethnomed. 2010, 6, 35. [CrossRef] [PubMed]

39. Luitel, D.R.; Rokaya, M.B.; Timsina, B.; Munzbergova, Z. Medicinal plants used by the Tamang community in the Makawanpur district of central Nepal. J. Ethnobiol. Ethnomed. 2014, 10, 5. [CrossRef] [PubMed]

40. Hasan, M.K.; Gatto, P.; Jha, P.K. Traditional uses of wild medicinal plants and their management practices in Nepal-A study in Makawanpur district. Int. J. Med. Aromat. Plants. 2013, 3, 102-112.

41. Malla, B.; Gauchan, D.P.; Chhetri, R.B. An ethnobotanical study of medicinal plants used by ethnic people in Parbat district of western Nepal. J. Ethnopharmacol. 2015, 165, 103-117. [CrossRef]

42. Manandhar, N.P. A survey of medicinal plants of Jajarkot district, Nepal. J. Ethnopharmacol. 1995, 48, 1-6. [CrossRef]

43. O'Neill, A.R.; Rana, S.K. An ethnobotanical analysis of parasitic plants (Parijibi) in the Nepal Himalaya. J. Ethnobiol. Ethnomed. 2016, 12, 14. [CrossRef]

44. Acharya, R.; Acharya, K.P. Ethnobotanical study of medicinal plants used by Tharu community of Parroha VDC, Rupandehi district, Nepal. Sci. World 2009, 7, 80-84. [CrossRef]

45. Kunwar, R.M.; Burlakoti, C.; Chowdhary, C.L.; Bussmann, R.W. Medicinal plants in farwest Nepal: Indigenous uses and pharmacological validity. Med. Aromat. Plant. Sci. Biotechnol. 2010, 4, 28-42.

46. Kunwar, R.M.; Adhikari, N. Ethnomedicine of Dolpa district, Nepal: the plants, their vernacular names and uses. Lyonia 2005, 8, 43-49.

47. Kunwar, R.M.; Uprety, Y.; Barlakoti, C.; Chowdhary, C.L.; Bussmann, R.W. Indigenous use and ethnopharmacology of medicinal plants in far-west Nepal. Ethnobot. Res. Appl. 2009, 7, 6-28. [CrossRef]

48. Rokaya, M.B.; Uprety, Y.; Poudel, R.C.; Timsina, B.; Munzbergova, Z.; Asselin, H.; Tiwari, A.; Shrestha, S.S.; Sigdel, S.R. Traditional uses of medicinal plants in gastrointestinal disorders in Nepal. J. Ethnopharmacol. 2014, 158 Pt A, 221-229. [CrossRef]

49. Bhattarai, S.; Chaudhary, R.P.; Taylor, R.S. Ethno-medicinal plants used by the people of Nawalparasi district, central Nepal. Our. Nat. 2009, 7, 82-99. [CrossRef]

50. Shrestha, N.; Shrestha, S.; Koju, L.; Shrestha, K.K.; Wang, Z. Medicinal plant diversity and traditional healing practices in eastern Nepal. J. Ethnopharmacol. 2016, 192, 292-301. [CrossRef] [PubMed]

51. Shrestha, P.M.; Dhillion, S.S. Medicinal plant diversity and use in the highlands of Dolakha district, Nepal. J. Ethnopharmacol. 2003, 86, 81-96. [CrossRef]

52. Singh, A.G. Medicinal plants as a source of antipyretic agent in Terai region of Western Nepal. Int. J. Appl. Sci. Biotechnol. 2013, 1, 118-126. [CrossRef]

53. Singh, A.G.; Kumar, A.; Tewari, D.D. An ethnobotanical survey of medicinal plants used in Terai forest of western Nepal. J. Ethnobiol. Ethnomed. 2012, 8, 19. [CrossRef] 
54. Vandebroek, I.; Damme, P.V.; Puyvelde, L.V.; Arrazola, S.; Kimpe, N.D. A comparison of traditional healers' medicinal plant knowledge in the Bolivian Andes and Amazon. Soc. Nat. Resour. 2004, 59, 837-849. [CrossRef] [PubMed]

55. Bennett, B.C. Plants and people of the Amazonian rainforests: the role of ethnobotany in sustainable development. Bioscience 1992, 42, 599-607. [CrossRef]

56. Abbas, Z.; Khan, S.M.; Alam, J.; Khan, S.W.; Abbasi, A.M. Medicinal plants used by inhabitants of the Shigar Valley, Baltistan region of Karakorum range-Pakistan. J. Ethnobiol. Ethnomed. 2017, 13, 53. [CrossRef] [PubMed]

57. Burlakoti, C.; Kunwar, R.M. Folk herbal medicines of Mahakali watershed area, Nepal. In Medicinal Plants in Nepal: An Anthology of Contemporary Research; Jha, P.K., Karmacharya, S.B., Chettri, M.K., Thapa, C.B., Shrestha, B.B., Eds.; Ecological Society (ECOS): Kathmandu, Nepal, 2008; pp. 187-193.

58. Singh, A.; Nautiyal, M.C.; Kunwar, R.M.; Bussmann, R.W. Ethnomedicinal plants used by local inhabitants of Jakholi block, Rudraprayag district, western Himalaya, India. J. Ethnobiol. Ethnomed. 2017, 13, 49. [CrossRef] [PubMed]

59. Thapa, S. Medico-ethnobotany of Magar community in Salija VDC of Parbat district, central Nepal. Our. Nat. 2012, 10, 176-190. [CrossRef]

60. Bhattarai, S.; Chaudhary, R.P.; Taylor, R.S. Ethnomedicinal plants used by the people of Manang district, central Nepal. J. Ethnobiol. Ethnomed. 2006, 2, 41. [CrossRef] [PubMed]

61. Srithi, K.; Balslev, H.; Wangpakapattanawong, P.; Srisanga, P.; Trisonthi, C. Medicinal plant knowledge and its erosion among the Mien (Yao) in northern Thailand. J. Ethnopharmacol. 2009, 123, 335-342. [CrossRef]

62. Ghimire, S.K.; Gimenez, O.; Pradel, R.; McKey, D.; Aumeeruddy-Thomas, Y. Demographic variation and population viability in a threatened Himalayan medicinal and aromatic herb Nardostachys grandiflora: Matrix modelling of harvesting effects in two contrasting habitats. J. Appl. Ecol. 2008, 45, 41-51. [CrossRef]

63. Acharya, R. Ethnobotanical study of medicinal plants of Resunga hill used by Magar community of Badagaun VDC, Gulmi district, Nepal. Sci. World 2012, 10, 54-65. [CrossRef]

64. Chekole, G. Ethnobotanical study of medicinal plants used against human ailments in Gubalafto District, Northern Ethiopia. J. Ethnobiol. Ethnomed. 2017, 13, 55. [CrossRef]

65. Maroyi, A. Diversity of use and local knowledge of wild and cultivated plants in the eastern Cape province, south Africa. J. Ethnobiol. Ethnomed. 2017, 13, 43. [CrossRef] [PubMed]

66. Yaseen, G.; Ahmad, M.; Zafar, M.; Sultana, S.; Kayani, S.; Cetto, A.A.; Shaheen, S. Traditional management of diabetes in Pakistan: Ethnobotanical investigation from traditional health practitioners. J. Ethnopharmacol. 2015, 174, 91-117. [CrossRef] [PubMed]

67. Stepp, J.R.; Moerman, D.E. The importance of weeds in ethnopharmacology. J. Ethnopharmacol. 2001, 75, 19-23. [CrossRef]

68. Bekalo, T.H.; Woodmatas, S.D.; Woldemariam, Z.A. An ethnobotanical study of medicinal plants used by local people in the lowlands of Konta Special Woreda, southern nations, nationalities and peoples regional state, Ethiopia. J. Ethnobiol. Ethnomed. 2009, 5, 26. [CrossRef] [PubMed]

69. Singh, A.G.; Hamal, J.P. Traditional Phytotherapy of some medicinal plants used by Tharu and Magar communities of western Nepal, against dermatological disorders. Sci. World 2013, 11, 81-89. [CrossRef]

70. Abbasi, A.M.; Khan, M.A.; Ahmed, M.; Zafar, M. Herbal medicines used to cure various ailments by the inhabitants of Abbottabad district, north west frontier province, Pakistan. Indian J. Tradit. Knowl. 2010, 9 , 175-183.

71. Bhattarai, N.K. Medical ethnobotany in the Karnali zone, Nepal. Econ. Bot. 1992, 46, 257-261. [CrossRef]

72. Sun, Q.; Yang, G.; Zhang, M.; Zhang, M.; Chen, S.; Chen, P. Effect of Huangshukuihua (Flos Abelmoschi Manihot) on diabetic nephropathy: a Meta-analysis. J. Tradit. Chin. Med. 2015, 35, 15-20. [CrossRef]

73. Singh, M.P.; Malla, S.B.; Rajbhandari, S.B.; Manandhar, A. Medicinal plants of Nepal-Retrospects and Prospects. Econ. Bot. 1979, 32, 185-198. [CrossRef]

74. Bhattarai, S.; Chaudhary, R.P.; Quave, C.L.; Taylor, R.S. The use of medicinal plants in the trans-Himalayan arid zone of Mustang district, Nepal. J. Ethnobiol. Ethnomed. 2010, 6, 14. [CrossRef] [PubMed]

75. Lama, Y.C.; Ghimire, S.K.; Aumeerudyy-Thomas, Y. Medicinal Plants of Dolpo: Amchis' Knowledge and Conservation; WWF Nepal Program: Kathmandu, Nepal, 2001.

76. Bhattarai, N.K. Folk Anthelmintic drugs of central Nepal. Int. J. Pharm. 1992, 30, 145-150. [CrossRef] 
77. Kunwar, R.M.; Bussmann, R.W. Medicinal plants and quantitative ethnomedicine: A case study from Baitadi and Darchula district, far-west Nepal. J. Nat. Hist. Mus. 2009, 24, 73-82. [CrossRef]

78. Rijal, A. Living knowledge of the healing plants: Ethno-phytotherapy in the Chepang communities from the Mid-Hills of Nepal. J. Ethnobiol. Ethnomed. 2008, 4, 23. [CrossRef] [PubMed]

79. Tariq, A.; Mussarat, S.; Adnan, M. Review on ethnomedicinal, phytochemical and pharmacological evidence of Himalayan anticancer plants. J. Ethnopharmacol. 2015, 164, 96-119. [CrossRef] [PubMed]

80. Gaire, B.P.; Subedi, L. Medicinal plant diverisity and their pharmacological aspects of Nepal Himalayas. Pharmacogn. J. 2011, 3, 6-17. [CrossRef]

81. Sansanelli, S.; Ferri, M.; Salinitro, M.; Tassoni, A. Ethnobotanical survey of wild food plants traditionally collected and consumed in the Middle Agri Valley (Basilicata region, southern Italy). J. Ethnobiol. Ethnomed. 2017, 13, 50. [CrossRef] [PubMed]

82. Kunwar, R.M.; Fadiman, M.; Cameron, M.; Bussmann, R.W.; Thapa-Magar, K.B.; Rimal, B.; Sapkota, P. Cross-cultural comparison of plant use knowledge in Baitadi and Darchula districts, Nepal Himalaya. J. Ethnobiol. Ethnomed. 2018, 14, 40. [CrossRef]

83. Begossi, A. Use of ecological methods in ehnobotany: diversity indices. Econ. Bot. 1996, 50, $280-289$. [CrossRef]

84. WHO. Health System in Nepal: Challenges and Strategic Options; World Health Organization: Lalitpur, Nepal, 2007.

85. Kristiansen, B.; Penninga, L.; Diernaes, J.E.F. Challenging cause of bullous eruption of the hands in the Arctic. BMJ Case Rep. 2018, 2018. [CrossRef]

86. Umair, M.; Altaf, M.; Bussmann, R.W.; Abbasi, A.M. Ethnomedicinal uses of the local flora in Chenab riverine area, Punjab province Pakistan. J. Ethnobiol. Ethnomed. 2019, 15, 7. [CrossRef]

87. Joshi, B.; Sah, G.; Basnet, B.; Bhatt, M.; Sharma, D.; Subedi, K.; Janardhan, P.; Malla, R. Phytochemical extraction and antimicrobial properties of different medicinal plants: Ocimum sanctum (Tulsi), Eugenia caryophyllata (clove), Achyranthes bidentata (Datiwan) and Azadirachta indica (Neem). J. Microbial. Antimicrob. 2011, 3, 1-7.

88. Devi, P.U.; Murugan, S.; Suja, S.; Selvi, S.; Chinnaswamy, P.; Vijayanand, E. Antibacterial, in vitro lipid per oxidation and phytochemical observation on Achyranthes bidentata Blume. Pak. J. Nutr. 2007, 6, 447-451. [CrossRef]

89. Pandey, V.; Agrawal, V.; Raghavendra, K.; Dash, A.P. Strong larvicidal activity of three species of Spilanthes (Akarkara) against malaria (Anopheles stephensi Liston, Anopheles culicifacies, species C) and filaria vector (Culex quinquefasciatus Say). Parasitol. Res. 2007, 102, 171-174. [CrossRef] [PubMed]

90. Shah, A.J.; Gilani, A.H. Bronchodilatory effect of Acorus calamus (Linn.) is mediated through multiple pathways. J. Ethnopharmacol. 2010, 131, 471-477. [CrossRef] [PubMed]

91. Rajput, S.B.; Tonge, M.B.; Karuppayil, S.M. An overview on traditional uses and pharmacological profile of Acorus calamus Linn. (Sweet flag) and other Acorus species. Phytomedicine 2014, 21, 268-276. [CrossRef] [PubMed]

92. Cellini, L.; Di Campli, E.; Masulli, M.; Di Bartolomeo, S.; Allocati, N. Inhibition of Helicobacter pylori by garlic extract (Allium sativum). FEMS Immunol. Med. Microbiol. 1996, 13, 273-277. [CrossRef] [PubMed]

93. Wang, Y.C. Medicinal plant activity on Helicobacter pylori related diseases. World J. Gastroenterol. 2014, 20, 10368-10382. [CrossRef] [PubMed]

94. Iimuro, M.; Shibata, H.; Kawamori, T.; Matsumoto, T.; Arakawa, T.; Sugimura, T.; Wakabayashi, K. Suppressive effects of garlic extract on Helicobacter pylori-induced gastritis in Mongolian gerbils. Cancer Lett. 2002, 187, 61-68. [CrossRef]

95. Nagori, B.P.; Solanki, R. Role of medicinal plants in wound healing. Res. J. Med. Plants 2011, 5, $392-405$. [CrossRef]

96. Hisam, E.E.; Zakaria, Z.A.; Mohtaruddin, N.; Rofiee, M.S.; Hamid, H.A.; Othman, F. Antiulcer activity of the chloroform extract of Bauhinia purpurea leaf. Pharm. Biol. 2012, 50, 1498-1507. [CrossRef]

97. Sakthivel, M.; Palani, P. Isolation, purification and characterization of antimicrobial protein from seedlings of Bauhinia purpurea L. Int. J. Biol. Macromol. 2016, 86, 390-401. [CrossRef] [PubMed]

98. Janbaz, K.H.; Gilani, A.H. Studies on preventive and curative effects of berberine on chemical-induced hepatotoxicity in rodents. Fitoterapia 2000, 71, 25-33. [CrossRef] 
99. Joshi, P.V.; Shirkhedkar, A.A.; Prakash, K.; Maheshwari, V.L. Antidiarrheal activity, chemical and toxicity profile of Berberis aristata. Pharm. Biol. 2011, 49, 94-100. [CrossRef] [PubMed]

100. Potdar, D.; Hirwani, R.R.; Dhulap, S. Phyto-chemical and pharmacological applications of Berberis aristata. Fitoterapia 2012, 83, 817-830. [CrossRef] [PubMed]

101. Singh, M.; Srivastava, S.; Rawat, A.K. Antimicrobial activities of Indian Berberis species. Fitoterapia 2007, 78, 574-576. [CrossRef] [PubMed]

102. Sohni, Y.R.; Kaimal, P.; Bhatt, R.M. Prophylactic therapy of Salmonella typhi septicemia in mice with a traditionally prescribed crude drug formulation. J. Ethnopharmacol. 1995, 45, 141-147. [CrossRef]

103. Gilani, A.U.H.; Janbaz, K.H. Preventive and curative effects of Berberis aristata Fruit extract on paracetamoland $\mathrm{CCl}_{4}$-induced hepatotoxicity. Phytother. Res. 1995, 9, 489-494. [CrossRef]

104. Neag, M.A.; Mocan, A.; Echeverria, J.; Pop, R.M.; Bocsan, C.I.; Crisan, G.; Buzoianu, A.D. Berberine: Botanical occurrence, traditional uses, extraction methods, and relevance in cardiovascular, metabolic, hepatic, and renal disorders. Front. Pharmacol. 2018, 9, 557. [CrossRef]

105. Somania, R.; Singhai, A.K.; Shivgunde, P.; Jain, D. Asparagus racemosus Willd (Liliaceae) ameliorates early diabetic nephropathy in STZ induced diabetic rats. Indian J. Exp. Biol. 2012, 50, 469-475.

106. Roy, D.C.; Barman, S.K.; Shaik, M.M. Current updates on Centella asiatica: phytochemistry, pharmacology and traditional uses. Med. Plant Res. 2013, 3, 20-26.

107. Cheng, C.L.; Koo, M.W. Effects of Centella asiatica on ethanol induced gastric mucosal lesions in rats. Life Sci. 2000, 67, 2647-2653. [CrossRef]

108. Sharma, C.; Mansoori, M.N.; Dixit, M.; Shukla, P.; Kumari, T.; Bhandari, S.P.; Narender, T.; Singh, D.; Arya, K.R. Ethanolic extract of Coelogyne cristata Lindley (Orchidaceae) and its compound coelogin promote osteoprotective activity in ovariectomized estrogen deficient mice. Phytomedicine 2014, 21, 1702-1707. [CrossRef] [PubMed]

109. Agarwal, R.; Gupta, S.K.; Srivastava, S.; Agrawal, S.S.; Saxena, R. Lowering of intraocular pressure by topical application of Daucus carota seed extract in rabbits. Indian. J. Exp. Biol. 2008, 46, 541-546. [PubMed]

110. Balasubramaniam, P.; Pari, L.; Menon, V.P. Protective Effect of Carrot (Daucus carota L.) Against Lindane-induced Hepatotoxicity in Rats. Phytother. Res. 1998, 12, 434-436. [CrossRef]

111. Bishayee, A.; Sarkar, A.; Chatterjee, M. Hepatoprotective activity of carrot (Daucus carota L.) against carbon tetrachloride intoxication in mouse liver. J. Ethnopharmacol. 1995, 47, 69-74. [CrossRef]

112. Singh, K.; Singh, N.; Chandy, A.; Manigauha, A. In vivo antioxidant and hepatoprotective activity of methanolic extracts of Daucus carota seeds in experimental animals. Asian Pac. J. Trop. Biomed. 2012, 2, 385-388. [CrossRef]

113. Mahmoudi, Z.; Soleimani, M.; Saidi, A.; Khamisipour, G.; Azizsoltani, A. Effects of Foeniculum vulgare ethanol extract on osteogenesis in human mecenchymal stem cells. Avicenna J. Phytomed. 2013, 3, 135-142.

114. Kim, T.H.; Kim, H.J.; Lee, S.H.; Kim, S.Y. Potent inhibitory effect of Foeniculum vulgare Miller extract on osteoclast differentiation and ovariectomy-induced bone loss. Int. J. Mol. Med. 2012, 29, 1053-1059. [CrossRef]

115. Dada, E.O.; Ekundayo, F.O.; Makanjuola, O.O. Antibacterial activites of Jatropha curcas (Linn) on Coliforms isolated from surface waters in Akure, Nigeria. Int. J. Biomed. Sci. 2014, 10, 25-30.

116. Rahman, M.M.; Ahmad, S.H.; Mohamed, M.T.M.; Rahman, M.Z.A. Antimicrobial compounds from leaf extracts of Jatropha curcas, Psidium guajava, and Andrographis paniculata. Sci. World J. 2014, 2014, 1-8.

117. Ahmad, S.; Wahid, M.A.; Bukhari, A.Q.S. Fungistatic action of Juglans. Antimicrob. Agents Chemother. 1973, 3, 436-438. [CrossRef] [PubMed]

118. Zhou, J.; Zhou, S.; Zeng, S. Experimental diabetes treated with trigonelline: Effect on beta cell and pancreatic oxidative parameters. Fundam. Clin. Pharmacol. 2013, 27, 279-287. [CrossRef] [PubMed]

119. Zhou, J.Y.; Zhou, S.W.; Zeng, S.Y.; Zhou, J.Y.; Jiang, M.J.; He, Y. Hypoglycemic and hypolipidemic effects of ethanolic extractof Mirabilis jalapa L. root on normal and diabetic mice. Evid. Based Complement. Altern. Med. 2012, 2012, 1-9.

120. Sasa, M.; Inoue, I.; Shinoda, Y.; Takahashi, S.; Seo, M.; Komoda, T.; Awata, T.; Katayama, S. Activating effect of momordin, extract of bitter melon (Momordica Charantia L.), on the promoter of human PPARdelta. J. Atheroscler. Thromb. 2009, 16, 888-892. [CrossRef] [PubMed]

121. Lokegaonkar, S.P.; Nabar, B.M. In vitro assessment of the antiplaque properties of crude M. alba leaf extract. J. Herb. Med. Toxicol. 2011, 5, 71-77. 
122. Islam, B.; Khan, S.N.; Haque, I.; Alam, M.; Mushfiq, M.; Khan, A.U. Novel anti-adherence activity of mulberry leaves: inhibition of Streptococcus mutans biofilm by 1-deoxynojirimycin isolated from Morus alba. J. Antimicrob. Chemother. 2008, 62, 751-757. [CrossRef] [PubMed]

123. Zuo, G.Y.; Yang, C.X.; Han, J.; Li, Y.Q.; Wang, G.C. Synergism of prenylflavonoids from Morus alba root bark against clinical MRSA isolates. Phytomedicine 2018, 39, 93-99. [CrossRef]

124. Park, K.M.; You, J.S.; Lee, H.Y.; Baek, N.I.; Hwang, J.K. Kuwanon G: an antibacterial agent from the root bark of Morus alba against oral pathogens. J. Ethnopharmacol. 2003, 84, 181-185. [CrossRef]

125. Sakat, S.S.; Tupe, P.; Juvekar, A. Gastroprotective effect of Oxalis Corniculata (whole plants) on experimentally induced gastric ulceration in Wistar rats. Ind. J. Pharm. Sci. 2012, 74, 48-53.

126. Ojewole, J.A. Hypoglycemic and hypotensive effects of Psidium guajava Linn. (Myrtaceae) leaf aqueous extract. Methods Find. Exp. Clin. Pharmacol. 2005, 27, 689-695. [CrossRef]

127. George, B.P.; Parimelazhagan, T.; Kumar, Y.T.; Sajeesh, T. Antitumor and Wound Healing Properties of Rubus ellipticus Smith. J. Acupunct. Meridian. Stud. 2015, 8, 134-141. [CrossRef] [PubMed]

128. Lutterodt, G.D.; Ismail, A.; Basheer, R.H.; Baharudin, H.M. Antimircobial effects of Psidium guajava extract as one mechanism of its antidiarrheal action. Malays. J. Med. Sci. 1999, 6, 17-20. [PubMed]

129. Abbas, S.R.; Sabir, S.M.; Ahmad, S.D.; Boligon, A.A.; Athayde, M.L. Phenolic profile, antioxidant potential and DNA damage protecting activity of sugarcane (Saccharum officinarum). Food Chem. 2014, 147, 10-16. [CrossRef] [PubMed]

130. Khan, S.W.; Tahir, M.; Lone, K.P.; Munir, B.; Latif, W. Protective effect of Saccharum officinarum L. (sugarcane) juice on Isoniazid induced hepatotoxicity in male Albino mice. J. Ayb. Med. Coll. Abbottabad. 2015, 27, 346-350.

131. Joshi, B.C.; Prakash, A.; Kalia, A.N. Hepatoprotective potential of antioxidant potent fraction from Urtica dioica Linn. (whole plant) in $\mathrm{CCl}_{4}$ challenged rats. Toxicol. Rep. 2015, 2, 1101-1110. [CrossRef] [PubMed]

132. Dubey, D.; Patnaik, R.; Ghosh, G.; Padhy, R.N. In vitro antibacterial activity, gas chromatography-mass spectrometry analysis of Woodfordia fruticosa Kurz. leaf extract and host toxicity testing with in vitro cultured lymphocytes from human umbilical cord blood. Osong Public Health Res. Perspect. 2014, 5, 298-312. [CrossRef] [PubMed]

133. Singh, G.; Singh, R.; Verma, P.K.; Singh, R.; Anan, A. Anthelmintic efficacy of aqueous extract of Zanthoxylum armatum DC. seeds against Haemonchus contortus of small ruminants. J. Parasit. Dis. 2016, 40, 528-532. [CrossRef] [PubMed]

134. Kim, T.H.; Kim, J.K.; Kang, Y.H.; Lee, J.Y.; Kang, I.J.; Lim, S.S. Aldose reductase inhibitory activity of compounds from Zea mays L. BioMed Res. Int. 2013, 2013, 727143. [CrossRef]

135. Giacosa, A.; Morazzoni, P.; Bombardelli, E.; Riva, A.; Bianchi Porro, G.; Rondanelli, M. Can nausea and vomiting be treated with ginger extract? Eur. Rev. Med. Pharmacol. Sci. 2015, 19, 1291-1296.

136. Manandhar, N.P. Plants and People of Nepal; Timber Press: Portland, OR, USA, 2002.

(C) 2019 by the authors. Licensee MDPI, Basel, Switzerland. This article is an open access article distributed under the terms and conditions of the Creative Commons Attribution (CC BY) license (http://creativecommons.org/licenses/by/4.0/). 